\title{
Imaging groundwater infiltration dynamics in the karst vadose zone with long-term ERT monitoring
}

\author{
Arnaud Watlet ${ }^{1,2, *}$, Olivier Kaufmann ${ }^{1}$, Antoine Triantafyllou ${ }^{1,3}$, Amaël Poulain ${ }^{4}$, Jonathan E. Chambers ${ }^{5}$, \\ Philip I. Meldrum ${ }^{5}$, Paul B. Wilkinson ${ }^{5}$, Vincent Hallet ${ }^{4}$, Yves Quinif ${ }^{1}$, Michel Van Ruymbeke ${ }^{2}$, and \\ Michel Van Camp ${ }^{2}$ \\ ${ }^{1}$ Geology and Applied Geology Unit, Faculty of Engineering, University of Mons, Place du Parc 20, 7000 Mons, Belgium \\ ${ }^{2}$ Seismology-Gravimetry, Royal Observatory of Belgium, Avenue Circulaire 3, 1180 Uccle, Belgium \\ ${ }^{3}$ Laboratoire de Planétologie et Géodynamique - Nantes (LPGN), UFR Sciences et Techniques, Université de Nantes, \\ UMR-CNRS 6112, Rue de la Houssinière 2, BP92208, 44322 Nantes CEDEX 3, France \\ ${ }^{4}$ Department of Geology, University of Namur, Rue de Bruxelles 61, 5000 Namur, Belgium \\ ${ }^{5}$ British Geological Survey, Environmental Science Centre, Keyworth, Nottingham NG12 5GG, UK \\ * Invited contribution by Arnaud Watlet, recipient of the EGU Hydrological Sciences \\ Outstanding Student Poster and PICO Award 2016.
}

Correspondence: Arnaud Watlet (arnaud.watlet@umons.ac.be)

Received: 1 August 2017 - Discussion started: 28 August 2017

Accepted: 17 January 2018 - Published: 1 March 2018

\begin{abstract}
Water infiltration and recharge processes in karst systems are complex and difficult to measure with conventional hydrological methods. In particular, temporarily saturated groundwater reservoirs hosted in the vadose zone can play a buffering role in water infiltration. This results from the pronounced porosity and permeability contrasts created by local karstification processes of carbonate rocks. Analyses of time-lapse 2-D geoelectrical imaging over a period of 3 years at the Rochefort Cave Laboratory (RCL) site in south Belgium highlight variable hydrodynamics in a karst vadose zone. This represents the first long-term and permanently installed electrical resistivity tomography (ERT) monitoring in a karst landscape. The collected data were compared to conventional hydrological measurements (drip discharge monitoring, soil moisture and water conductivity data sets) and a detailed structural analysis of the local geological structures providing a thorough understanding of the groundwater infiltration. Seasonal changes affect all the imaged areas leading to increases in resistivity in spring and summer attributed to enhanced evapotranspiration, whereas winter is characterised by a general decrease in resistivity associated with a groundwater recharge of the vadose zone. Three types of hydrological dynamics, corresponding to areas with distinct lithological and structural features, could be identified via changes
\end{abstract}

in resistivity: (D1) upper conductive layers, associated with clay-rich soil and epikarst, showing the highest variability related to weather conditions; (D2) deeper and more resistive limestone areas, characterised by variable degrees of porosity and clay contents, hence showing more diffuse seasonal variations; and (D3) a conductive fractured zone associated with damped seasonal dynamics, while showing a great variability similar to that of the upper layers in response to rainfall events. This study provides detailed images of the sources of drip discharge spots traditionally monitored in caves and aims to support modelling approaches of karst hydrological processes.

\section{Introduction}

Karst regions provide drinking water for a quarter of the world's population (Ford and Williams, 2007; Mangin, 1975). In a changing world, improving the management of vital resources is a key problem, as highlighted in Hartmann et al. (2014). Achieving enhanced management calls for a better understanding of superficial water movements, which are known to be strongly heterogeneous in karst areas. The autogenic recharge of the phreatic zone of karst 
aquifers is driven by water infiltration through the vadose zone (White, 2002). The thickness of this vadose zone varies from one karst system to another but is commonly described as two entities: (i) its uppermost layer, the soil joined with the so-called epikarst which is characterised by high weathering and porosity of carbonate rocks, overlaying the (ii) infiltration zone. The hydrological function of both layers differs from one type of karst to another (e.g. Mediterranean or humid, young or mature karst landscapes; Klimchouk, 2004). While rainfall can directly feed the infiltration zone through sinkholes or open cracks in the epikarst, a part of meteoric water remains delayed in the epikarst (Bakalowicz, 2005). Locally, water can be stored in perched saturated pockets because of strong permeability contrasts with regard to lower layers. Such epikarst storage was proven to be sustainable enough to host aquatic biota (Sket et al., 2004) or to induce strong dilution of rainwater isotopic signatures (Perrin et al., 2003). In some regions, especially in China, such storage in the subsurface is expected to be great enough to sustainably provide water to populations (Williams, 2008). Nevertheless, these water reservoirs are likely to be seasonally influenced and laterally heterogeneous, interacting with the soil and biosphere through evapotranspiration, while seeping under gravity, or by overflow after intense rainfall events (Clemens et al., 1999; Goldscheider and Drew, 2007; Sheffer et al., 2011). Such leakage down to the infiltration zone therefore ranges from very slow seepages within the carbonated matrix porosity to quick flows through fractures and cracks in the carbonate rocks (Atkinson, 1977; Smart and Friederich, 1987).

All models describing karst hydrology agree on the dichotomy of matrix and conduit recharge processes (Hartmann et al., 2014). Karstification is expected to act on the porosity of the bulk rock and therefore on its hydraulic conductivity (Kiraly, 2003). Permanent storage in the vadose zone, responsible for perennial dripping recorded in cave networks, has been confirmed in several case studies (e.g. Arbel et al., 2010). However, compared to the epikarst, the role of the infiltration zone itself in delaying the infiltration and potentially storing groundwater in the matrix porosity remains an open question. In dry periods, dripping with unvarying low volume discharges is only explained by infiltration via low capacity routes or perched aquifers slowly releasing water into the underlying layers (Smart and Friederich, 1987). Hartmann et al. (2013) modelled the recharge of matrix reservoirs in the vadose zone by lateral exchange with saturated conduits. This confirms the possibility for these processes to occur at several levels within the infiltration zone, making it possible for groundwater to be stored not only in the epikarst, but in several subsystems of the entire vadose zone.

To support hydrological models, investigation techniques commonly consist of tracer tests or spring flow monitoring, mainly applied to the characterisation of the saturated zone but also tested in the vadose zone for the monitoring of stalactites drip discharge (e.g. Pronk et al., 2009). In particular, such experiments can provide evidence of variable transfer types. Natural caves provide great opportunities to study the vadose zone hydrodynamics from the inside with punctual and direct measurements and/or monitoring. Hydrographs or hydrochemical monitoring are often a valuable source of information. Although novel promising approaches for building dense cave drip discharge monitoring networks are rising (e.g. Mahmud et al., 2016, 2018), strong heterogeneities of karst areas often make it challenging to build robust networks that adequately capture groundwater storage variations in the vadose zone. Karst subsurface remains poorly known and not often instrumented or monitored. In particular, very little has been achieved to image and monitor perched reservoirs.

Geophysical methods provide non-invasive and integrated tools that can strongly improve karst hydrological knowledge. Hence, numerous studies have been conducted to characterise karst subsurface (see Chalikakis et al.; 2011, for a review). In terms of hydrological monitoring, Valois et al. (2011) and Deville et al. (2012) highlighted the signal of epikarst storage variations in gravity anomalies of repeated gravity measurements. Fores (2016) supported similar measurements with seismic noise monitoring.

In parallel, ERT (electrical resistivity tomography) monitoring methods have proved to be highly efficient, especially in hydrogeophysics (e.g. Coscia et al., 2012; Kuras et al., 2009; Revil et al., 2012) and in engineering and geotechnics for monitoring landslide areas (e.g. Chambers et al., 2013; Uhlemann et al., 2016a), contaminated sites (e.g. Caterina et al., 2017; Kuras et al., 2016; LaBrecque et al., 1996a) or permafrost regions (e.g. Supper et al., 2014). The strength of such methods resides in their effectiveness to track changes in the electrical properties of the subsurface, reflecting variations in moisture content, groundwater content, temperature or chemical properties. Binley et al. (2015) identify ERT monitoring as a key technique in the advancing of hydrogeophysical methods applicable for investigating subsurface processes. A few studies have already used repeated ERT surveys to track hydrological changes in karst areas. Recently, Xu et al. (2017) investigated time-lapse ERT data to define subsurface characteristics near the Lascaux cave (France). Carrière et al. (2016) successfully used time-lapse ERT and magnetic resonance sounding (MRS) to identify the role of the porous matrix in regulating water infiltration from epikarst structures, previously identified by groundpenetrating radar (GPR) and ERT surveys in southern France (Carrière et al., 2013). Meyerhoff et al. (2012) applied repeated time-lapse ERT measurements to visualise variations in karst saturated conduits' conductivity, assessing the mixing of matrix water and surface water. In parallel, Kaufmann and Deceuster (2014) have demonstrated the applicability of using ERT to image the porous matrix associated with karstification processes. Altogether, these studies demonstrate the applicability of such techniques with regard to hydrological purposes in karst, although they spotted real challenges: the heterogeneity of the subsurface making the interpreta- 
tion of resistivity models more complex and the difficulty of practically ensuring proper contacts for electrodes, especially in the presence of outcropping limestone (Chalikakis et al., 2011).

To the best of our knowledge, this paper presents the first attempt of long-term, permanently installed, and high spatialand temporal-resolution ERT monitoring of karst subsurface hydrodynamics. Our experiment covers a 3-year monitoring period of the Rochefort site, a karst area located in south Belgium. The ERT measurements focus on a 2-D profile and comprise two sub-periods: a first 3-month period of daily ERT measurements started in April 2014 and a second 2-year series of almost uninterrupted measurements from March 2015. Additional hydrological data such as moisture probes and in-cave percolating water discharge measurements support the experiment. The monitoring site focuses on a small part of the karst area, at the entrance of the Lorette cave. Such a local-scale approach supports the need to study karst hydrology on all scales (Hartmann, 2016) to build extensive data sets available for strengthening hydrological models.

\section{Description of the Rochefort karst system}

The study area is located over the central part of the Lorette cave, next to the city of Rochefort in southern Belgium. The Lorette cave is one of several cavities that belong to the Wamme-Lomme karst system (Marion et al., 2011), a $10 \mathrm{~km}$ long karst area located in the Calestienne, a band of outcropping Devonian limestone crossing southern Belgium ENE following the Variscan fold-and-thrust belt (Fig. 1c; Pirson et al., 2008). These units host the most widespread karsts and caving systems of Belgium (Willems and Ek, 2011). They can be summarised as two main units: the Charlemont limestone, which includes four limestone formations; and the Fromelennes limestone, at the bottom of which shales of the Flohimont member act as an impervious layer, hydrogeologically speaking. The Wamme-Lomme karst system itself results from the cross-cutting of the Lomme river, $5 \mathrm{~km}$ north-east of Rochefort, and its main tributary, the Wamme river, with the Calestienne units. The system ends when the Lomme river meets the shales and limestones $5 \mathrm{~km}$ southwest of Rochefort, at the Eprave resurgence (Fig. 1a).

In the Lorette cave and on a larger scale, in the Rochefort area, limestone layers are part of an overturned syncline (Fig. 1b) comprising the Charlemont limestone strata striking N070 with a moderate to high dipping value of $50^{\circ}$ to the SSE (Vandycke and Quinif, 2001). All of them are situated within the same lithostratigraphic formation (the Mont d'Haurs Formation) and form alternating series of decimetric well-preserved limestone and weathered and porous limestone strata with occasional thin clay interbeds.
The study site is part of the Rochefort Cave Laboratory (RCL) (Camelbeeck et al., 2011; Quinif et al., 1997), located in the central part of the Lorette cave in an underground area that covers about 1 ha at the surface (Fig. 1c). Most of the area, located at $\sim 225 \mathrm{~m}$ above ordnance datum (AOD) on a limestone plateau, slopes gently towards the Lomme valley, which lies about $165 \mathrm{~m}$ AOD. A large sinkhole (typical collapse depression of karst regions) of $\sim 25 \mathrm{~m}$ of diameter and $\sim 20 \mathrm{~m}$ deep gives access to the Lorette cave. This cave is characterised by a well-developed karst network (Vandycke and Quinif, 2001) comprising large passages with diameters of several metres that follow the strike direction of the stratigraphic unit (N070), as well as smaller conduits normal to the main ones (Fig. 1c). The Val d'Enfer room, which is in direct connection with the entrance sinkhole, forms the largest feature of the Lorette cave where several limestone layers outcrop. The northernmost gallery is another site of interest where structures of the massif are visible. Most of the galleries of the RCL are located between $\sim 180$ and $\sim 190 \mathrm{~m}$ AOD, i.e. 40 to $30 \mathrm{~m}$ from the surface.

In terms of hydrogeology, in low water conditions, the water table shows up in the Lorette cave at $\sim 162 \mathrm{~m} \mathrm{AOD}$ at the end of a steep small conduit, which is about $60 \mathrm{~m}$ below the surface of the plateau. A tiny underground river, with an average discharge of $\sim 50 \mathrm{~m}^{3} \mathrm{~s}^{-1}$ (Poulain et al., 2015a), is also accessible at some points. These accesses to the phreatic zone allow monitoring the water table levels with CTD (conductivity temperature depth) divers. Due to Belgium's temperate maritime climate, the Rochefort region experiences mean annual precipitation of $890 \mathrm{~mm}$ distributed all over the year with monthly averages ranging from 60 to $82 \mathrm{~mm}$, as calculated by the Belgian Royal Meteorological Institute over the last 30 years. Hence, the infiltration reaches its maximum in winter while evapotranspiration predominates in summer. Heavy rainfall periods, intense storms or snow melting periods increase the runoff, swelling the rivers, which causes flash floods to occur in the caves of the system. Flash flood events may temporarily raise the saturated zone to a maximum of 174 m AOD (Van Camp et al., 2006; Watlet et al., $2018 b$ ). In such cases, the main cavities of the RCL area, which are located above this level, remain dry.

\section{Environmental monitoring}

At the surface of the RCL site, a small building, located at the border of the large sinkhole, hosts the instruments data loggers and the resistivity meter. The eastern part of the site is mostly asphalted, with a parking area and two minor roads, while the rest of the area is wooded, including the sinkhole where the ERT profile is installed (Fig. 1c). The underground part of the RCL site benefits from infrastructures, such as steps and paths originally built for a former touristic exploitation of the cave in the beginning of the 20th century. Some of 

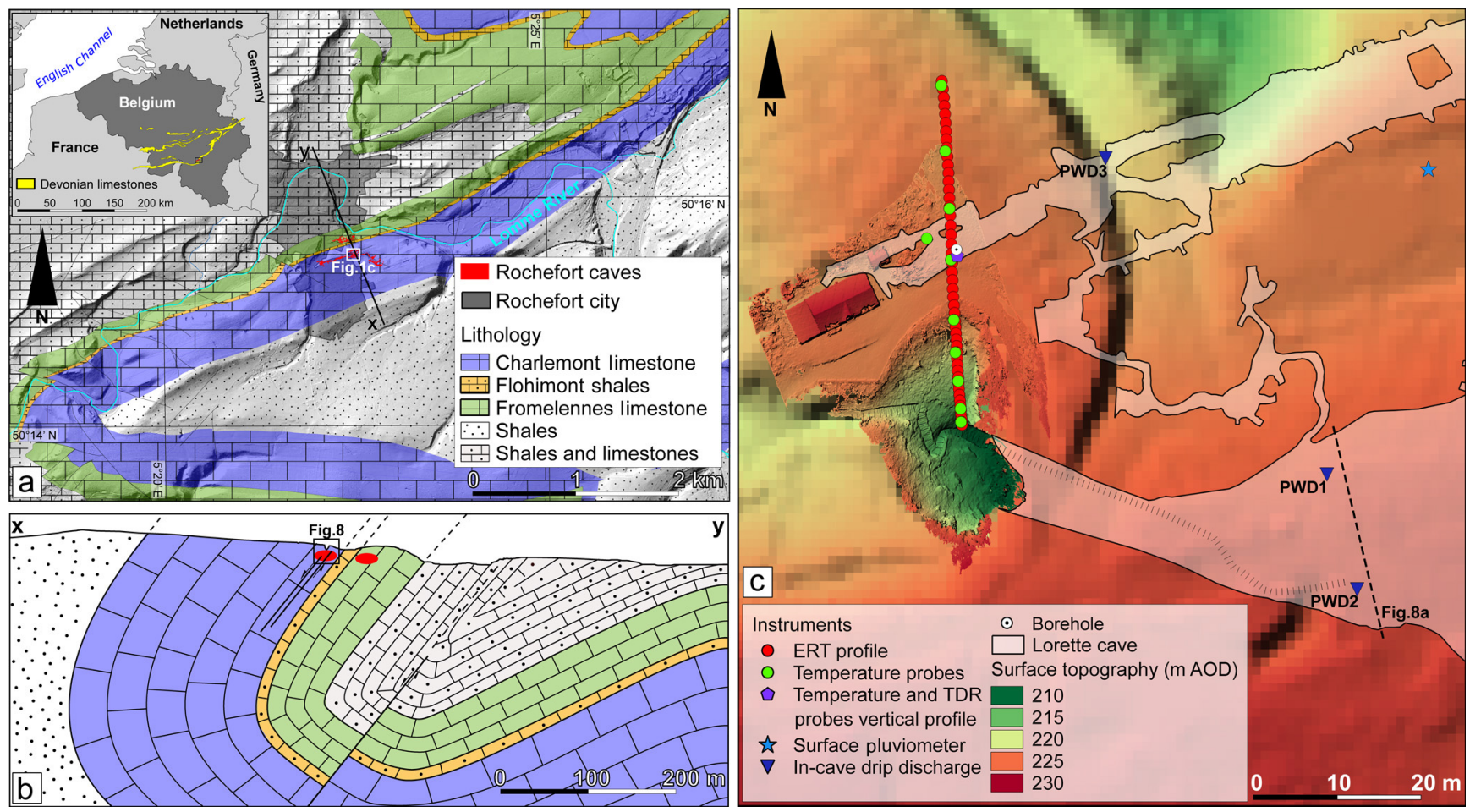

Figure 1. (a) Simplified geological map of the Rochefort area (after Barchy et al., 2014), highlighting the limestone formations and the Rochefort caves. (b) Geological cross section ( $x$ to $y$ in 1A), modified after Delvaux de Fenffe (1987). It displays the overturned syncline marked by high dipping sedimentary layers (N070-S50) in the Rochefort caves area as well as active normal faults evidenced by Vandycke and Quinif (2001). (c) General overview of the Rochefort monitoring site. Electrodes from the ERT profile, WCR and temperature probes as well as the rain gauge are shown. Background $1 \mathrm{~m}$ digital elevation model (based on lidar data of the Public Service of Wallonia) is replaced by a high-resolution surface topography model from a 3-D photoscan (Triantafyllou et al., 2016). This highlights the monitored sinkhole that provides access to the Lorette cave (in white).

these infrastructures have been secured against collapse for our study.

\subsection{Sensor network installation}

Several environmental sensors have been installed at the RCL site: soil moisture probes, in-cave percolating water gauges, and rain and percolating water conductivity probes. They are intended to support the ERT measurements. First, a vertical profile of five water content reflectometers (WCRs) from Campbell Instruments (CS616) is installed $2 \mathrm{~m}$ away from the ERT monitoring profile. They provide data with a resolution of $0.1 \%$ in volumetric water content (VWC) and a sensor variability of 0.5 and $1.5 \%$ VWC in dry and humid conditions respectively. The probes are inserted at depths of $10,30,50,75$ and $105 \mathrm{~cm}$ and have been operational since May 2015. Their sampling rate was $1 \mathrm{~h}$ for the first months of measurement and was changed to 1 min afterwards. As the average soil thickness is only $40 \mathrm{~cm}$ at the RCL site, a portion of fractured and weathered limestone mixed with clays and roots needed to be excavated to $105 \mathrm{~cm}$. This material was replaced after each WCR was installed. They are therefore surrounded by a mixture of limestone blocks clays and soil materials. Such heterogeneous materials make the calibration of the WCRs rather challenging, with the porosity $(\phi)$ of the soil and rocks surrounding each probe being hard to assess. Some assumptions on the porosity around the probes based on maximum thresholds reached during the monitoring period could however be proposed to estimate the saturation $(S=\mathrm{VWC} / \phi)$.

Additionally, the Lorette cave is equipped with percolation discharge monitoring concentrated in three specific locations. Two drip discharge gauges are installed in the Val d'Enfer room, one of which (PWD1, percolation water discharge station 1) monitors flows dripping through a subvertical open fracture oriented N160 in a clayey limestone layer, with the other one (PWD2) being installed under a karstified area where drips come out of a particularly porous limestone layer. The third station (PWD3) monitors one stalactite built on a massive limestone layer associated with very slow discharge in the northernmost passage at the vertical of the ERT profile. This area is generally much drier than the Val d'Enfer room. The thickness between the surface and the monitored inlet flows is $\sim 25 \mathrm{~m}$ for PWD1 and PWD2 and $\sim 33 \mathrm{~m}$ for PWD3. PWD1 and PWD3 have been monitored since 2001, 
but PWD1 suffered from instrumental problems from 2013 to 2015, when the instrument was replaced. The complete network was finalised in March 2016 with the additional PWD3 installed in the framework of this study. Measuring drip discharge is usually complex as calcite deposits can perturb the instruments, while the great variability of flow regimes is particularly challenging for the instrumental design. PWD1 and PWD2 are made of an auto-siphoning gauge with capacitive sensors designed by University of Mons, based on an original prototype from the Royal Observatory of Belgium (Kaufmann et al., 2016). The dripping water is collected in an inverted cone feeding a small upper tank which in turn feeds a larger lower tank. Capacitive sensors are plunged in each tank and return high frequency FM signals. This sampling allows the emptying of the tanks to be counted to estimate the flow rates. Using a small and a large tank increases the range of flows supported by the system $\left(0.5\right.$ to $\left.100 \mathrm{Lh}^{-1}\right)$. Since time resolution depends on the discharge, an interpolation is required to get a constant time step of $10 \mathrm{~min}$. The PWD3 instrument only comprises one capacitive sensor surrounding the tip of the monitored stalactite. The growing water drop creates a decrease in the FM signal, followed by a sharp increase triggered by the drop's fall.

Specific electrical conductivity $(\mathrm{SpC})$ measurements are also performed in-cave at the PWD1 monitoring station, as well as at the surface for monitoring rainwater conductivity, next to the ERT profile. Both measurements are performed using a Campbell CS547A probe (accuracy of $\pm 5 \%$ ).

Rainfall was monitored for the whole period of ERT monitoring using a Lufft tipping bucket type rain gauge with a 1 min sample rate, located on the RCL site itself. The locations of the WCR profile and rain gauge are shown in Fig. 1c. Additional potential evapotranspiration $\left(\mathrm{ET}_{0}\right)$ data are also available. $\mathrm{ET}_{0}$ is derived from the Penman-Monteith relationship (Allen et al., 1998) based on data from a meteorological station (Pameseb) located $5 \mathrm{~km}$ from the Rochefort monitoring site.

\subsection{Environmental data results}

Figure 2 shows the rainfall and $\mathrm{ET}_{0}$ data (a) in comparison with the soil moisture recorded by the WCR (b). This gives an overview of the climatic conditions experienced during the ERT monitoring experiment. The year 2015 can be considered as normal in terms of weather conditions, with rainfall homogeneously distributed, except for a short dry period in September 2015. ET $_{0}$ also follows expected trends, resulting in negative values of effective rainfalls during summer (given by rainfall - $\mathrm{ET}_{0}$ ). In comparison, 2014 and 2016 were more unusual, experiencing particularly wet summers, leading to very few periods of negative effective rainfall. This means that at least the uppermost layer was continuously fed by rainwater, which should result in high average moisture contents, as was monitored by the WCR for the 2016 period. However, a remarkable dry period affected southern Belgium at the end of summer 2016 (from 6 August to 15 October), with only $53 \mathrm{~mm}$ of precipitation. This is extremely low compared to the seasonal average for the area which normally equals $172 \mathrm{~mm}$ for the same period of time, based on the seasonal averages provided by the Belgian Royal Meteorological Institute. This period will be particularly interesting to look at with the ERT monitoring, as the lowest VWC measured at the site was reached in the top layers. Particularly high resistivity values are to be expected in the surface layer during that period. In particular, comparing ERT data from summer 2015 and the end of summer 2016 will be useful in identifying the role of $\mathrm{ET}_{0}$ and rainfall in the moisture contents of deeper layers.

Overall, soil moisture data inform on the dynamics of the infiltration at the location of the vertical profile, showing repeated rainfall infiltration processes. Every significant precipitation event progressively infiltrates the soil layer, producing a sharp increase in VWC followed by an exponential recession curve. The delay between the beginning of the rainfall event and the first arrival of infiltrating water depends on the intensity of the rainfall event, evapotranspiration conditions, the depth of the moisture probe and hydraulic conductivity parameters defining the soil retention curve. In winter, a delay of $\sim 14 \mathrm{~h}$ is observed between the 10 and $105 \mathrm{~cm}$ deep probe. In summer, this delay can be significantly longer, up to several days. The influence of evapotranspiration is clearly noticeable as fewer peaks are present in the VWC data set. After long droughts, such as that of August and September 2016, a delay of 85 days is noticed between the first moisture content peak observed at $10 \mathrm{~cm}$ depth and that observed at $105 \mathrm{~cm}$.

In parallel, in-cave percolating water discharge data bring crucial information on the infiltration processes occurring in the vadose zone at the RCL site. The three stations show different discharge dynamics given their location and the type of inlet flow that they sample. Smart and Friederich (1987) developed a drip discharge classification based on the relationships between maximum discharges and coefficients of variation of the discharge; they can be described as vadose flows for PWD1 and PWD2 and seepage flow for PWD3 respectively. Vadose flows refer to high discharges, albeit lower than for shaft flows, with a high variability, especially regarding their rainfall events responses. Seepage flows exhibit significantly lower discharges with low coefficients of variation but noticeable seasonal changes. Despite being classified as vadose flow, the PWD2 data set exhibits a strong seasonal pattern. It actually samples more of a dripping zone rather than one single inlet flow associated with one stalagmite or fracture. This could lead to overestimating the maximum discharge regarding the approach of Smart and Friederich (1987). PWD2 could therefore be described as seasonal drip, which differs from seepage flows by its higher coefficient of variation, following the modification of the classification after Baker (1997). The spatial proximity of 

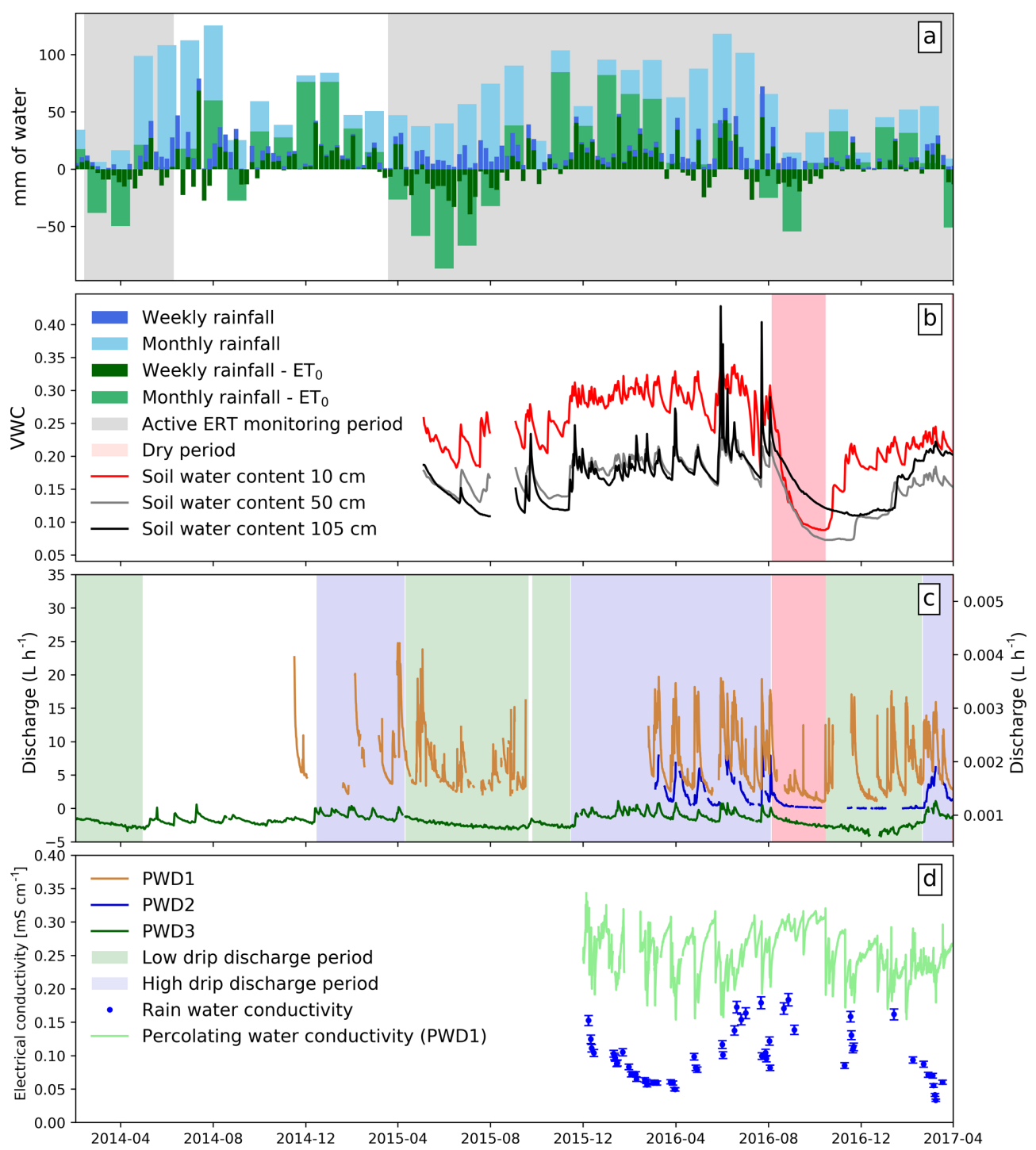

Figure 2. (a) Weekly and monthly rainfall as well as effective rainfall (estimated as rainfall $-\mathrm{ET}_{0}$ ). Shaded areas represent the periods with active ERT monitoring. (b) Three of the five WCR probe data sets for the 10, 50 and $105 \mathrm{~cm}$ depths. The long drought of August and September 2016 is highlighted in pale red. (c) Data set for the three percolation water discharge (PWD) stations. Periods of low discharge and high discharge are highlighted based on values from the PWD3 and PWD2 when available. (d) Electrical conductivity of rain water (for rainfall event $>5 \mathrm{~mm}$ ) and percolation water measured at the PWD1 station. Error bars stand for the instrumental error announced by the manufacturer $( \pm 5 \%)$.

PWD1 and PWD2 exhibiting different discharge regimes testifies to the high heterogeneity of the Rochefort karst.

Seasonal cycles affecting percolating water are strongly related to effective rainfall and soil moisture data, as shown in Fig. 2. Similar observations have been described and analysed in multiple studies, highlighting the buffering role of the epikarst in water infiltration (e.g. Genty and Deflandre, 1998; Poulain et al., 2015b; Sheffer et al., 2011; Aquilina et al., 2003). Arbel et al. (2010) distinguish perennial drip discharge, which explains the bottom threshold visible in PWD2 and PWD3 in summer (Fig. 2c), and seasonal drips that stop during summer and are characterised by longer re- cession times. Additionally, post-storm drips directly follow rainfall events and decay after a few weeks, exhibiting a high discharge variability. The first two types can be related to diffuse flow that propagates through the matrix, while the last type refers to quick flows and conduit infiltrations (Hartmann et al., 2014; Lange et al., 2010; Perrin et al., 2003). Overall, these classifications highlight the duality of water infiltration and recharge in karst systems.

Unlike PWD2 and PWD3, PWD1 does not exhibit a clear seasonal trend, even though the baseflow threshold and post-storm drip decrease during the driest periods, while longer recession curves are observed. This is especially the 
case for the August and September 2016 drought. Poulain et al. (2018) provides a specific analysis of the diffuse flow and quick-flow components of PWD1, supported by a vadose dye tracing test. It confirms the two-flow regime as a mixing of matrix and conduit infiltration. PWD2 and PWD3 seem to depend more on diffuse flow through the matrix but a part of quick flow is still present in the signal. In conclusion, drip discharge data reflect well their station's location: PWD1 samples inlet flows from an open fracture crosscutting a clayey limestone layer, which explains the great quick-flow component from post-storm drip type percolation through the fracture. PWD2 monitors drip discharge from a porous limestone layer - water coming out directly from the rock matrix, without the presence of stalactites. Finally, PWD3 is installed on a dry location and samples inlet flows from a stalactite built on a massive limestone layer. This explains its very low observed perennial drip discharge described as seepage flow.

The electrical conductivity of the percolating water (Fig. 2d) displays some variations following rainfall events and related recharge processes, but no seasonal trends are evidenced. The observed values average $0.25 \mathrm{mS} \mathrm{cm}^{-1}$ $(40 \Omega \mathrm{m})$, while maximum values of $0.33 \mathrm{mS} \mathrm{cm}^{-1}(30 \Omega \mathrm{m})$ are recorded after long droughts. Rainfall events result in rapid decreases in the electrical conductivity which sometimes dip to $0.15 \mathrm{mS} \mathrm{cm}^{-1}(65 \Omega \mathrm{m})$. These values are attributed to rain water rapidly infiltrating conduits, mixed with more conductive groundwater. The $0.33 \mathrm{mS} \mathrm{cm}^{-1}$ threshold is believed to account for the pore-water maximum electrical conductivity in the subsurface of the RCL site, whereas recharge processes due to rainfall tend to result in decreased electrical conductivity. Electrical conductivity of the rain water is also monitored at the surface ranging from 0.20 to $0.04 \mathrm{mS} \mathrm{cm}^{-1}$ (50 to $250 \Omega \mathrm{m}$ ). Given the very small delay between rainfall events and in-cave discharge increases, the significant difference between the minimum electrical conductivity measured for rain water $\left(0.04 \mathrm{mS} \mathrm{cm}^{-1}\right)$ and dripping water $\left(0.15 \mathrm{mS} \mathrm{cm}^{-1}\right)$ testifies to rapid mixing processes with groundwater and/or efficient ionic leaching by the percolation water. Such rapid changes are in accordance with findings of Hunkeler and Mudry (2007). Poulain et al. (2018) also provide a study of the relationship between the discharge flows and the electrical conductivity at the RCL site.

In summary, results of the environmental monitoring of the vadose zone already bring valuable information on the infiltration processes occurring at the RCL site that will be useful for guiding the interpretation of the geophysical monitoring. Overall, hydrological seasonal trends are already discernible from these data sets, while different infiltration dynamics attributed to rainfall events are observed, illustrating the heterogeneity of the karst subsurface.

\section{$4 \quad$ ERT monitoring}

\subsection{ERT monitoring installation}

A preliminary study was necessary to assess the feasibility of ERT monitoring at the RCL site and more specifically to define the most appropriate location for installing the electrodes permanently. Seven ERT surveys around the RCL site were therefore conducted in 2013, which constituted an important step for the design of the experiment. They resulted in identifying the sinkhole giving access to the cave as an area with heterogeneous electrical resistivity features likely to be of interest for monitoring complex hydrological processes. A profile of 48 electrodes, with a pronounced topography, was therefore installed permanently. Twenty-eight electrodes from this profile are set at the top of the limestone massif and 20 others along the slope of the sinkhole (Fig. 1c). Most of the electrodes are buried 20 to $30 \mathrm{~cm}$ below the surface and made of stainless steel hollow tubes with diameters of $2 \mathrm{~cm}$ and lengths of $12 \mathrm{~cm}$ (for total surface of contact of $\sim 150 \mathrm{~cm}^{2}$ ) (see photos in Fig. A1). Good electrical contact of each tube with the soil was ensured with bentonite. Because a limestone stratum is outcropping at the bottom of the sinkhole, the five southernmost electrodes of the profile are directly bolted into the rock. A stainless steel wedge anchor was used to fix a $100 \mathrm{~cm}^{2}$ stainless steel plate to the rock (total surface of $\sim 110 \mathrm{~cm}^{2}$ ). A protective cap made of polyurethane foam covers each electrode in order to reduce corrosion processes and for safety reasons. The electrode spacing was chosen to be $1 \mathrm{~m}$, as recommended amongst others by Clément et al. (2009) to monitor shallow recharge processes.

Two acquisition systems were installed at the RCL site. A first testing period lasted from March 2014 until June 2014. An automated time-lapse electrical resistivity tomography (ALERT) acquisition system developed by the British Geological Survey (Kuras et al., 2009) collected daily dipole-dipole (DD) measurements. After this testing period, we installed a four-channel Iris Syscal Pro resistivity meter in March 2015, which is still presently measuring. Daily multiple gradient (GD) and DD data were collected, except for summer 2015 and winter 2016 where DD arrays, which require higher injection power, suffered from battery malfunction issues. Both acquisition systems are remotely controlled from the office. Data are automatically sent to a server and checked for measurement errors. The acquisition system is installed in a brick shelter, furnished with a wired internet connection and a 230 VAC power access, providing ideal infrastructure for an ERT monitoring site.

The measurement protocols involve dipole-dipole and multiple gradient types. They were chosen because of their effectiveness for multichannel data acquisition purposes as well as their good image resolution capabilities (Dahlin and Zhou, 2004). On the one hand, DD arrays are well suited to image lateral features and allow efficient collection of 
reciprocal measurements. Exchanging current and potential electrodes should ideally deliver the same results, as stated by the reciprocity theorem (Parasnis, 1988). Comparing forward and reciprocal measurements provides a robust method for estimating the data error and quality (LaBrecque et al., 1996b; Wilkinson et al., 2012). The DD type surveys chosen in this experiment use dipole lengths ( $a$ factor) of 1 to $3 \mathrm{~m}$ and dipole separation ( $n$ factor) of $1 a$ to $10 a$ and involve reciprocal measurements. On the other hand, GD arrays are asymmetrical and especially suited for multichannel acquisition as long as no reciprocal measurements are acquired. Unlike the normal measurements, reciprocal GD measurements are indeed practically unsuitable for multi-receiver acquisition, leading to long acquisition duration time, which increases the risk of real changes occurring during a measurement sequence. However, GD arrays are characterised by a better signal-to-noise ratio than the DD arrays (Dahlin and Zhou, 2006). GD type surveys chosen for this experiment use a combination of dipole separation ( $a$ factor) of 1 to $4 \mathrm{~m}$ and a current-electrode separation ( $s$ factor) of $1 a$ to $4 a$ (for further information on multiple-gradient arrays, see Dahlin and Zhou, 2006). Although this configuration provides a lower depth of investigation than that of the chosen DD array, it has an improved resolution for shallow depth. As previously mentioned, acquiring daily reciprocal GD measurements would be too time consuming; only normal GD measurements were performed for time-lapse monitoring.

\subsection{ERT data processing}

\subsubsection{Data processing, quality control and error estimation}

We developed a semi-automated workflow involving routines for data acquisition, storage, filtering, inversion and visualisation. A first data filtering is applied on the repeatability error of each measurement. During the acquisition, the potential difference on the measurement dipole of each quadrupole is measured two to four times by the resistivity meter. Distributions of the repeatability error are shown in Fig. 3a. For DD arrays, data having repeatability with a SD (repeatability or stacking error) over $5 \%$, as well as measured potentials lower than $1 \mathrm{mV}$, are automatically filtered. Following this step, reciprocal errors are computed for the DD type data set.

Reciprocal error is the resistance difference between normal and reciprocal measurement, i.e. when current injection and potential dipoles are swapped. Figure $3 b$ shows the distribution of the reciprocal errors for the whole DD type data set, after filtering for repeatability errors and low potentials. Data with relative reciprocal errors over $20 \%$ were also removed. Reciprocal errors are used as a noise estimate for the inversion procedure, where the resistance of each measurement needs to be weighted. Overall, filtering on repeatability error and reciprocal error leads to $15 \%$ of all the DD measurements being rejected, mainly due to too-low mea- sured potentials. GD type surveys have no reciprocal measurement available for each daily data set. A punctual reciprocity test was performed on GD arrays and showed relative reciprocal errors slightly higher than those of DD arrays. This is attributed to the fact that GD surveys have a measured resistances range significantly broader than that of the DD surveys. Furthermore, the signal-to-noise ratio shows a slightly different order of magnitude between normal and reciprocal measurements in case of GD surveys, which is expected to increase the reciprocal error. Another possible explanation for this is that real changes may occur during the GD surveys. Since the GD reciprocals take longer to measure (single channel) than the DD reciprocals, there is more time for greater changes to occur, leading to greater differences between forward and reciprocal measurements. This also explains why the DD reciprocal errors are greater than the DD repeatability error: stacking measurements are measured close together in time, but forward and reciprocal pairs are separated by larger times. Given that GD arrays have no reciprocal measurements available for the entire monitoring period, the repeatability error filtering threshold was set down to $0.5 \%$, which also takes into account the lower mean of the repeatability error distribution compared to that of the DD arrays, as visible in Fig. 3a. Following this filtering, only $1.5 \%$ of GD measurements were rejected.

Contact resistances along the ERT profile also showed high temporal variations, following the moisture conditions at the site (Fig. 4a). The high clay content of the soil at RCL ensures a very good electrode-ground contact in humid conditions. However, it favours shrinking during dry periods that can reduce the surface contact of electrodes with surrounding soil materials. Such processes therefore increase the contact resistances that, in turn, are a source of increased measurement errors. Higher contact resistances are usually noticed as they produce greater repeatability errors and reciprocal errors. In dry periods, this leads to a higher number of rejected data after filtering. In August 2016, electrode \#12, placed in the middle of the slope of the sinkhole, started to show significantly bad contact resistances $(>50 \mathrm{k} \Omega$ ) which induced poor measurement quality. For time-lapse processing, all the quadrupoles comprising electrode \#12 were therefore continuously rejected, which reduced the maximum number of measurements per survey and the resolution in that part of the ERT profile. Full surveys are composed of 990 DD reciprocal measurements (901 without electrode \#12) and 1420 GD measurements (1296 without electrode \#12). Figure 4b and c summarise the percentage of rejected measurements per survey for the monitoring period. Days with more than $10 \%$ of rejected data were removed from the time-lapse data set and are therefore not shown in Fig. $4 \mathrm{~b}$ and c. Given the greater amount of rejected DD measurements, this results in $467 \mathrm{DD}$ and 588 GD data sets.

In December 2015, despite the fact that the monitoring site was equipped with AC power supply, the injection batteries started to fail because of the increased power demanded 

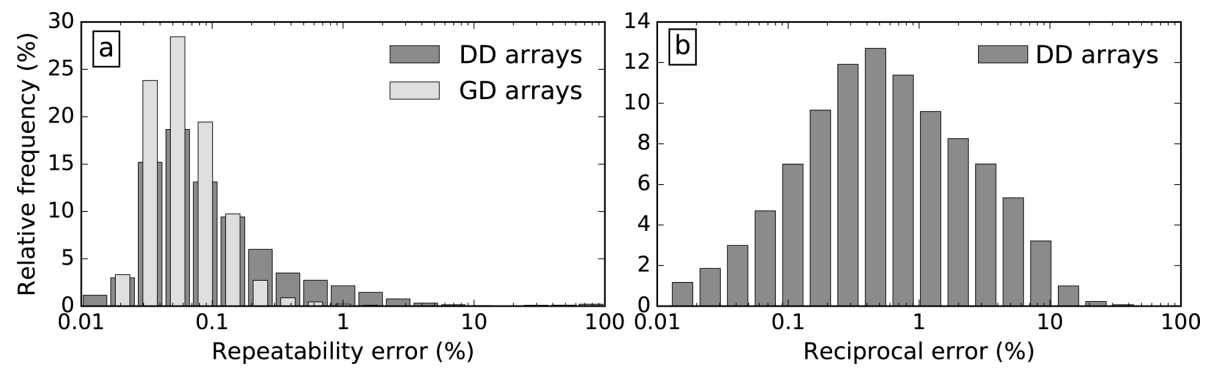

Figure 3. Relative frequency of (a) the repeatability (stacking) error for the whole GD and DD data sets, and (b) the reciprocal error for the whole DD data set.

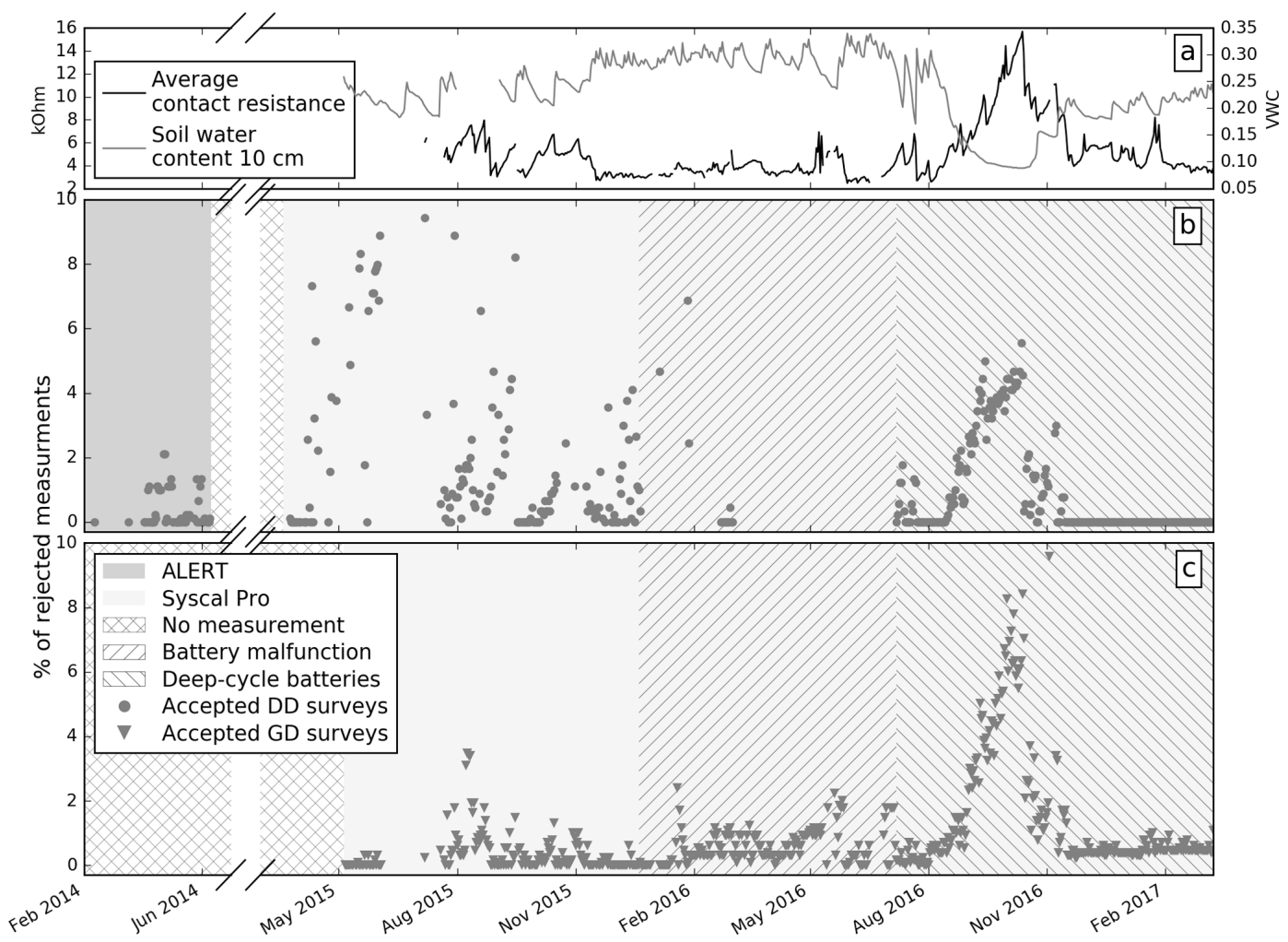

Figure 4. (a) Evolution of the contact resistances averaged along the ERT profile and the soil water content of the $10 \mathrm{~cm}$ probe (see point 2.6). (b) DD surveys used for the study, after filtering of the available data sets. Each data set with more than $10 \%$ of rejected data (less than 810 accepted reciprocal measurements) was removed. (c) GD surveys used for the study, after filtering of the available data sets. Each data set with more than $10 \%$ of data rejected (less than 1180 accepted measurements) was removed. Note the break in the $x$ axis between June 2014 and March 2015 where no measurements were acquired. Malfunction of the battery recharge caused most of the DD available data sets to be rejected between mid-December 2015 and the end of June 2016. The drying period in August and September 2016 also lead to a greater number of rejected data. The lower maximum measurement per survey after August 2016 is due to bad contact of electrode \#12.

by the resistivity meter to deliver higher voltages, especially for DD surveys. The battery malfunction led to the rejection of almost all the DD surveys acquired during that period. GD surveys were less sensitive to the problem because the power and voltage requirements were lower on average. The greater percentage of rejected data in 2015 is also attributed to the batteries being slightly less efficient for repeated highvoltage injections. The original starting type batteries were replaced by deep-cycle gel batteries in July 2016, especially designed for deep discharge, similar to those frequently used with solar panels. The long drying period from August to October 2016 is responsible for a progressive increase in the amount of rejected data for both DD and GD surveys.

Temperature variations of the subsurface can have significant impacts on resistivity data (Brunet et al., 2010). Because a marked temperature gradient is expected in the sinkhole, we modelled the 2-D temperature field using the framework of pyGIMLi (Rücker et al., 2017; www.pygimli.org) 
using data from a network of eight temperature probes installed along the ERT profile and a $105 \mathrm{~cm}$ vertical profile of five temperature probes. Correcting for the effect of temperature in time-lapse ERT can be implemented in several ways. We chose to apply the method described in Hayley (2007) in which both the data and the modelled resistivities are corrected for temperature effects. Details on the temperature correction applied in this study are available in Supplement Sect. S1.

\subsubsection{Time-lapse inversion}

Data are inverted with Boundless Electrical Resistivity Tomography software (BERT), which is based on a finite element modelling (Günther et al., 2006; Rücker et al., 2006). Each of the DD and GD data sets after filtering and correction for temperature effects constitutes one data set for the inversion, whereas the inverted results of the first data set of the DD and GD series constitute the reference model for the whole time series. The inversion is carried out using a $L_{1}$ (robust) data constraint to mitigate bias produced by outlying data on the convergence of the inversion and a $L_{2}$ (smoothness) model constraint. The time-lapse inversion procedure comprises an additional time regularisation constraint $\left(\lambda_{t}=50\right)$ for the inversion of each subsequent data set, linking each of the inverted models to the reference model. This is used to smooth over time the inverted results between each other, while facilitating the convergence of each inversion. Note that the structural assumptions presented in Sect. 5 are not used as model constraints in the inversion. This choice was made because of the uncertainty concerning the spatial continuity of structural observations in such a heterogeneous karst context. Time-lapse inversion achieved acceptable convergence between the observed data and the reconstructed model data as average RMS misfits of $11.7 \%$ (SD $1.2 \%$ ) and $2.4 \%$ (SD $0.2 \%$ ) for DD and GD arrays were respectively retrieved. Higher RMS errors of DD arrays are not really surprising. They are attributed to the lower signal-to-noise ratios of dipole-dipole arrays and the greater number of large dipoles configurations in the chosen DD protocol. The chi-squared test is another way for evaluating the convergence of the inversion. A chi-squared value about 1 generally indicates that the error model is appropriately calibrated and does not lead to data underfitting or overfitting. Time-lapse inversion resulted in chi-squared values of 0.87 (SD 0.1) and 0.84 (SD 0.1) for DD and GD time series respectively.

BERT provides a coverage parameter calculated from the sum of the absolute sensitivities of the measurements. Areas with high coverage are better constrained by the measurements and the modelling choices than low coverage areas. The coverage is usually incorporated in the data visualisation as a transparency mask that is used to weight the data depending on their associated coverage values.

\subsection{ERT imaging results}

\subsubsection{Resistivity distribution}

The inverted resistivity images of DD and GD arrays show a great dispersion. Images from DD arrays have a better coverage at depth than those of GD arrays. Hence, they are able to identify deeper structures, while GD arrays have a better resolution at shallow depth. In such a karst context, massive limestone layers are expected to be highly resistive $(>1000 \Omega \mathrm{m})$. This means that conductive anomalies can point to areas of higher clay or moisture content. Figure $5 \mathrm{a}$ presents an image after inversion of the DD array of May 2015, which corresponds to a period of average weather conditions. Most areas of the image are resistive $(>1000 \Omega \mathrm{m})$. A 1 to $3 \mathrm{~m}$ thick, highly conductive layer $(<200 \Omega \mathrm{m})$ is present at the surface of the limestone plateau (A in Fig. 5a). This layer progressively disappears along the slope of the sinkhole. The northern half of the ERT image also shows a highly resistive $(>3000 \Omega \mathrm{m})$ area below the surface layer (B). A second conductive zone (C), dipping $\sim 70^{\circ}$ to the north, is revealed in the central part of the image and corresponds with the topography inflexion. Resistivity values of that area are comparable with those of the surface layer. To the south, additional thin conductive layers appear at the surface (D) and 3 to $5 \mathrm{~m}$ below the surface of the slope (E), under a more resistive thin layer. The rest of the image shows high resistivities up to $5000 \Omega \mathrm{m}$. Results of the GD array (Fig. 5b) for the same period also image the conductive layer at the surface, the high resistivity area in the northern part of the survey and the thin conductive layer below a thin more resistive layer in the slope. The highly conductive zone dipping steeply to the north in the middle of the DD image result is not reconstructed at depth on this section. This is not surprising as configurations of the GD protocol lead to lower sensitivities at depth.

Field observations corroborate the presence of the conductive layer on the plateau, as this area is characterised by a $\sim 40 \mathrm{~cm}$ clay-rich soil layer. This layer becomes progressively thinner in the slope of the sinkhole and disappears at the sixth electrode from the bottom, which required the first six electrodes to be attached to the outcropping rock, as previously mentioned. The conductive layer at the top of the plateau is thicker than $40 \mathrm{~cm}$. Even if a blurring effect is expected from the smoothness constraints, this layer seems to be about 1 to $3 \mathrm{~m}$ thick. It is interpreted as the soil and epikarst, where the porosity is higher than in deeper limestone layers.

The high resistivity values may correspond to those of reasonably non-karstified limestone. Intermediate values may indicate karstified limestone with a low clay or moisture content. Such values could also result from clay-rich interbeds between limestone strata. Given the resolution of the ERT image, such thin features cannot be precisely delineated. In the case of a highly conductive thin shape surrounded by 


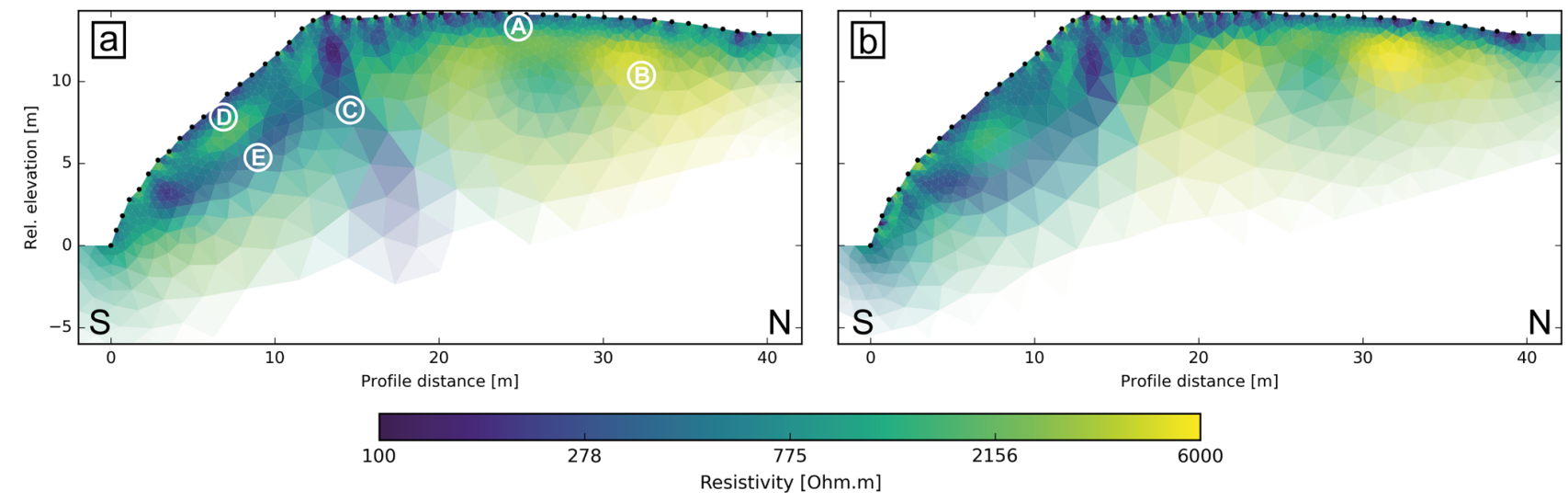

Figure 5. Image of the inverted resistivities for DD (a) and GD (b) data sets in average weather conditions (March 2017). Letters (A-D) indicate anomalies described in the main text.

highly resistive materials, the inversion process could only build a larger conductive area with an intermediate resistivity value. The strongly dipping conductive feature that crosses the resistivity image in the middle also deserves discussion. It could represent a fractured zone either filled with clays or characterised by a higher moisture content than the surrounding materials. The time-lapse imaging will give some insights on the role of this part of the section in hydrogeological processes taking place in the near surface by highlighting changes in resistivity through the year.

\subsubsection{Time-lapse imaging}

There are several ways to visualise spatiotemporal changes in resistivity resulting from a time-lapse ERT inversion, such as computing resistivity ratios, log of resistivity ratios or percentage of change in resistivity, or log of resistivity. The basis on which the time-lapse comparison is made must also be appropriately assessed. It may involve visualisation of resistivity variations that occur between each data set, highlighting sharp resistivity changes. Lower frequency resistivity variations usually need to be visualised as changes with respect to a baseline. Such a baseline does not necessarily have to be the same as the reference model used in the time-lapse inversion process. In many studies, the baseline used to track resistivity changes consists of resistivity measurements performed before the process to be monitored begins. In some cases, this stage is easy to assess. For instance, when monitoring artificial water injection, the baseline commonly consists of an ERT survey made prior to the start of the injection (e.g. Robert et al., 2012). In other cases, including this one, a clear baseline is more difficult to assess because the beginning of the process of interest, i.e. recharge processes, is not clearly identifiable. Here, the seasonality of the vadose zone moisture conditions (Fig. 2) complicates the choice of a clear baseline to visualise the time-lapse resistivity images.
As this study focuses on groundwater recharge, a baseline in dry conditions was chosen. The long drought of August to mid-October 2016 during which WCR moisture measurements dropped dramatically (Fig. 2) corresponds to the driest conditions encountered during the monitoring period. ERT surveys of 15 October 2016 corresponding to the last day of this drought were therefore chosen as baselines $\left(\rho_{0}\right)$. Consequently, change in log of resistivity with respect to that baseline is computed (Fig. 6b) for each data set $\left(\rho_{i}\right)$ as

$\Delta \rho=\left(\frac{\log \rho_{i}}{\log \rho_{0}}-1\right) \times 100$.

Our preference was for change in log of resistivity because it gives a better overview of gradual spatial variation than changes in absolute resistivity.

Figure $6 \mathrm{~b}$ shows changes in log resistivity for 10 of the 467 DD data sets (see the Supplement for an animation of the resistivity variations for the whole time series of the DD and GD data sets). Although this kind of display highlights broad variations in resistivity, it assumes that the nature of the underground materials would give comparable resistivity changes if exposed to similar moisture conditions. This is usually the case in fairly homogeneous environments, whereas it is not necessarily valid in highly heterogeneous contexts. Such visualisation could therefore overlook subtle changes in regions associated with resistivity variations of smaller amplitudes. To address that problem, our approach consists of displaying images of normalised resistivity $\left(\rho_{\text {norm }}\right)$ rescaled between 0 and 1 (Fig. 6c), such as for each cell $(c)$ of the mesh:

$\rho_{\text {norm }}^{c}=\frac{\rho_{i}^{c}-\rho_{\min }^{c}}{\rho_{\max }^{c}-\rho_{\min }^{c}}$,

where $\rho_{i}^{c}$ corresponds to the resistivity of a cell at a time step $i$, while $\rho_{\max }^{c}$ and $\rho_{\min }^{c}$ are respectively the minimum and maximum resistivity value of each cell over the whole time 

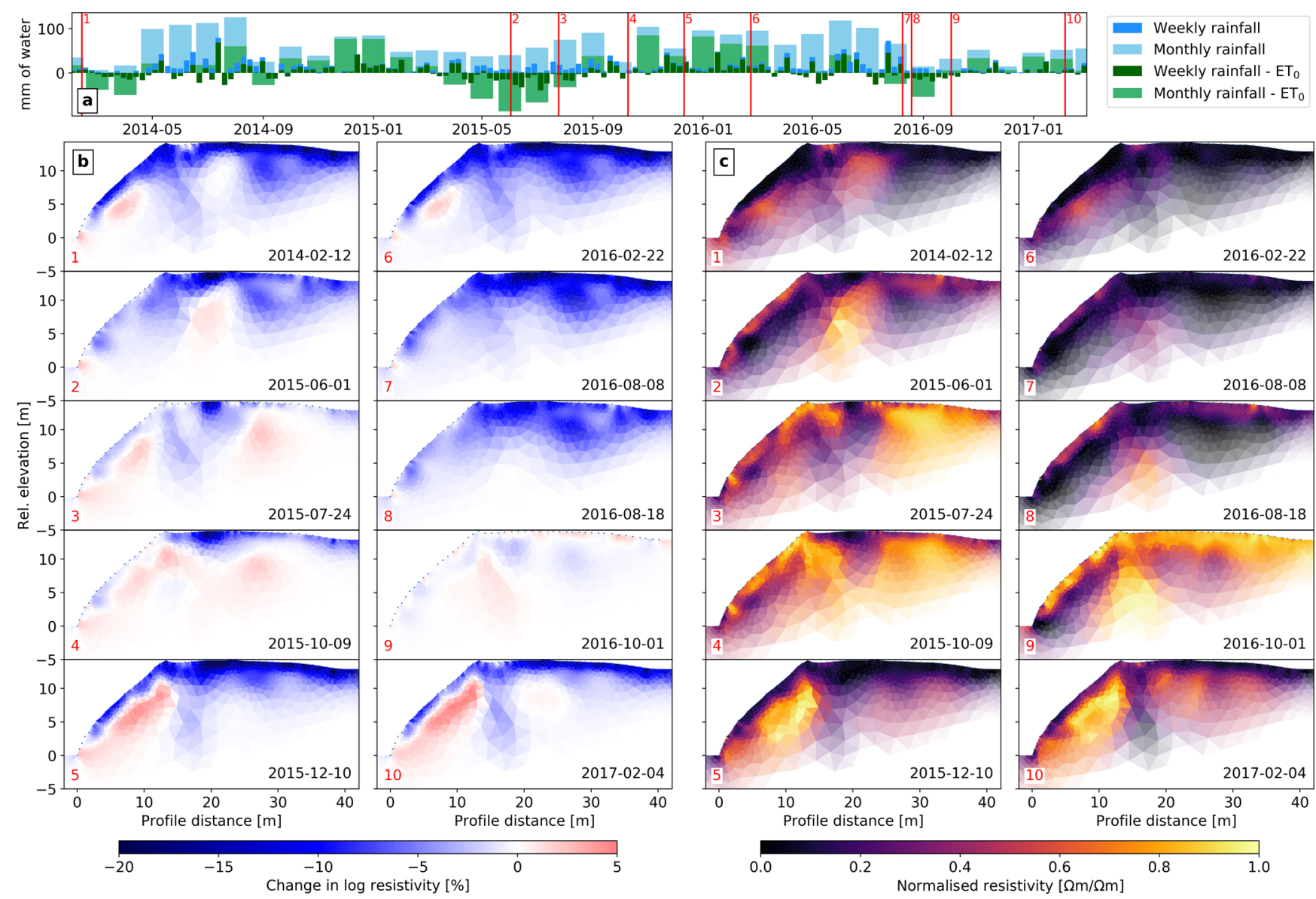

Figure 6. (a) Rainfall and effective rainfall. (b) Changes in log resistivity (see Eq. 1) for 10 of the 467 DD data sets, as highlighted in Fig. 6a. (c) Normalised resistivity (see Eq. 2) for the same data sets. See the Supplement for an animation of the resistivity variations for the whole time series of the DD and GD data sets.

series. In this way it is possible to interpret the resistivity of each cell with reference to its variation in the considered time span.

Overall, changes in log resistivity compared to the driest conditions encountered at the surface during the whole monitoring period show that the majority of the variation in resistivity is negative. This means that most of the subsurface experienced a decrease in resistivity, consistent with an increase in the moisture. The area most affected by intense ( $>10 \%$ ) decreases in resistivity is the surface of the plateau. A surface layered structure $(\sim 2.5 \mathrm{~m}$ deep $)$ can be seen to vary temporally with higher amplitudes than the rest of the model. It corresponds to the conductive layer displayed in Fig. 5. Highest negative changes in resistivity are reached during winter (December to March). The basis of this layer does not follow perfectly the surface topography, going a little bit deeper at the north and south of the plateau. In particular, in the middle of the profile, in the southern side of the plateau, a small area ( $\sim 4 \mathrm{~m}$ in extent) remains at very negative values for a longer time. This area therefore seems to act as a small basin in terms of ground moisture, highlighting the very high spatial heterogeneity expected in a karst medium.

Below this surface layer, changes in log resistivity progressively attenuate, in the area of the higher resistivities imaged in Fig. 5, except for a circular shape discernible in the middle part of the plateau (centred around $5 \mathrm{~m}$ deep). This area that corresponds with the lower resistivity anomaly in Fig. 5 also experiences high negative resistivity variations in winter while showing slight increases in resistivity in summer compared to the driest condition at the surface. Such a behaviour in summer is also noticeable in deeper parts of the sinkhole area that are associated with low resistivity values. However, in winter resistivity changes remain less negative in that area.

The changes in the surface layer of the sinkhole follow the same dynamic as that of the surface of the plateau, except that it shows less intense log resistivity decrease $(<10 \%)$. The feature strongly dipping to the south, starting at the inflection of the slope of the sinkhole (associated with high conductivity in Fig. 5), also has a dynamic in terms of resistivity changes discernible from its surrounding areas. It 
shows mostly negative variations, smaller than those of the surface layer.

Figure $6 c$ comprises images of the normalised resistivities for the same time steps as those of Fig. 6b. In addition to providing images with an enhanced contrast, it allows clarification of the temporal signature of some regions of the image, especially the deeper ones. For example, the strongly dipping feature has a relatively low resistivity in the image of 24 July 2015 (Fig. 6b-3) that equals negative changes in surface layers of both the sinkhole and the plateau. When comparing that information with the normalised resistivity image (Fig. 6c-3), it appears that this strongly dipping feature displays normalised resistivity values $(\sim 0.2)$ closer to their measured minimum than those of the surface layers $(\sim 0.6)$. This means that for this time step, the dipping feature is very close to the maximum measured negative resistivity change while surface layers are in average conditions with respect to the resistivity distribution observed during the monitoring period. Similar observations can be made for the deep circular feature in the middle of the plateau for time steps of 18 August 2016 and 1st October 2016 (Fig. 6b-8 and b-9) where negative variations of resistivity similar to some of the surface layers and surrounding areas are not equally represented by the normalised resistivity images (Fig. 6c-8 and c-9). The deep area of the slope of the sinkhole is also interesting to look at in time steps of 1 June 2015, 8 August 2016 and 18 August 2016 (Fig. 6b-2, b-7 and b-8), as little change in resistivity relative to the minimum status can be seen in terms of normalised resistivity (Fig. 6c-2, c-7 and c-8).

In conclusion, this highlights the fact that several subsurface regions of the ERT profile experience pronounced changes in resistivity through the monitoring experiment period. These regions have their own resistivity signatures, as illustrated in Fig. 5, which testifies to the substantial heterogeneity inherent to karst systems. To simplify the observations, Fig. 7 clusters the recovered resistivities of the subsurface of the ERT profile in eight distinct regions that display different spatial and temporal resistivity signatures, based on their average resistivity values and arbitrary thresholds. It differentiates superficial layers (1, 2, 3 in Fig. 7) and deep regions $(4,5,6,7,8$ in Fig. 7). Interestingly, these regions display different dynamics in terms of the temporal resistivity evolution in Fig. 6. In particular, dynamics of the surface layers look dissociated from those of three deeper regions: the moderately resistive circular feature in the middle of the plateau (5), the deep layer in the slope of the sinkhole area (7) and the high dipping feature (8). The rest of the image does not show much noticeable change (6) except low variations in the rest of the deep parts of the plateau in the right side of the image (4). Table 1 summarises the temporal evolution of each region through time, highlighting their distinct characteristics.

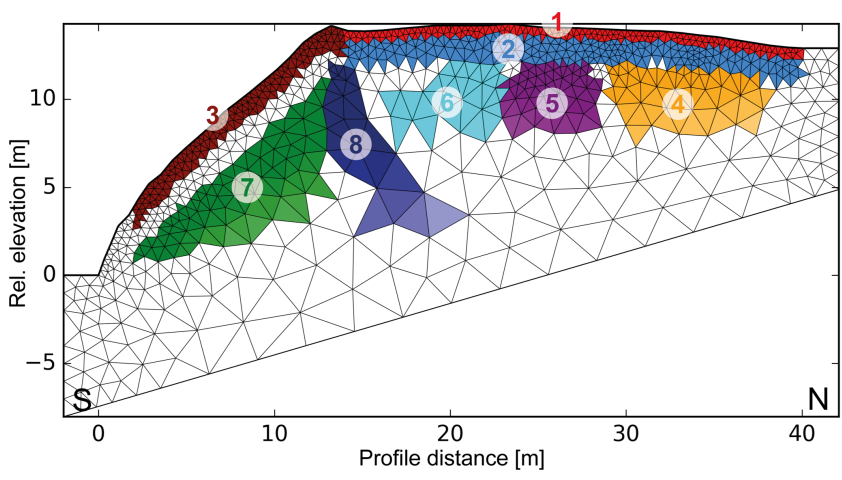

Figure 7. Subdivision of the ERT image in eight distinct regions based on their average resistivity values and arbitrary thresholds. Table 1 presents characteristics of the temporal evolution associated with each region.

Table 1. Statistics of the temporal evolution of the median of the absolute resistivity values for the eight regions defined in Fig. 7. For the values of each region, the table shows the temporal mean, median, standard deviation (SD) and SD normalised to the temporal mean resistivity. GD data sets are used for superficial regions (1, 2, 3 in Fig. 7) given the better resolution of that protocol at shallow depths. Resistivity values of the deeper regions $(4,5,6,7,8)$ come from the DD surveys, because of the greater imaging resolution at depth.

\begin{tabular}{lrrrrrrrr}
\hline & 1 & 2 & 3 & 4 & 5 & 6 & 7 & 8 \\
\hline Mean $(\Omega \mathrm{m})$ & 857 & 2327 & 851 & 4691 & 3003 & 3140 & 490 & 441 \\
Median $(\Omega \mathrm{m})$ & 708 & 2122 & 844 & 4713 & 3102 & 3253 & 492 & 438 \\
SD $(\Omega \mathrm{m})$ & 424 & 972 & 251 & 1025 & 753 & 536 & 51 & 63 \\
Normalised SD $(-)$ & 0.50 & 0.42 & 0.29 & 0.22 & 0.25 & 0.17 & 0.10 & 0.14 \\
\hline
\end{tabular}

\section{Micro-structural observations}

\subsection{Field, borehole and photoscan surveys}

As bedding planes, open joints, fractures and small conduits play an important role in water infiltration in karst systems, we investigated the local geological structures of the limestone massif, based on previous works from Vandycke and Quinif (2001) and Camelbeeck et al. (2011). These authors reported (Vandycke and Quinif, 2001) and monitored (Camelbeeck et al., 2011) active faults striking N070 (subparallel to the bedding foliations in the Lorette cave) with a recent normal slip combined with a small sinistral strikeslip component (see Fig. 1b). The relatively small scale of the monitoring area makes it relevant to study in detail the geological structure and lithology of the site. New observations from multiple sources have been gathered to build a lithological model of the monitoring site, comprising a field survey and the acquisition of a 3-D model of the RCL's main chamber, the Val d'Enfer room. This 3-D model results from a drone photoscan of the cavity and allows automatic detection of the orientations of planar structures and hence a statistical analysis of the main geological structures (i.e. sedimen- 


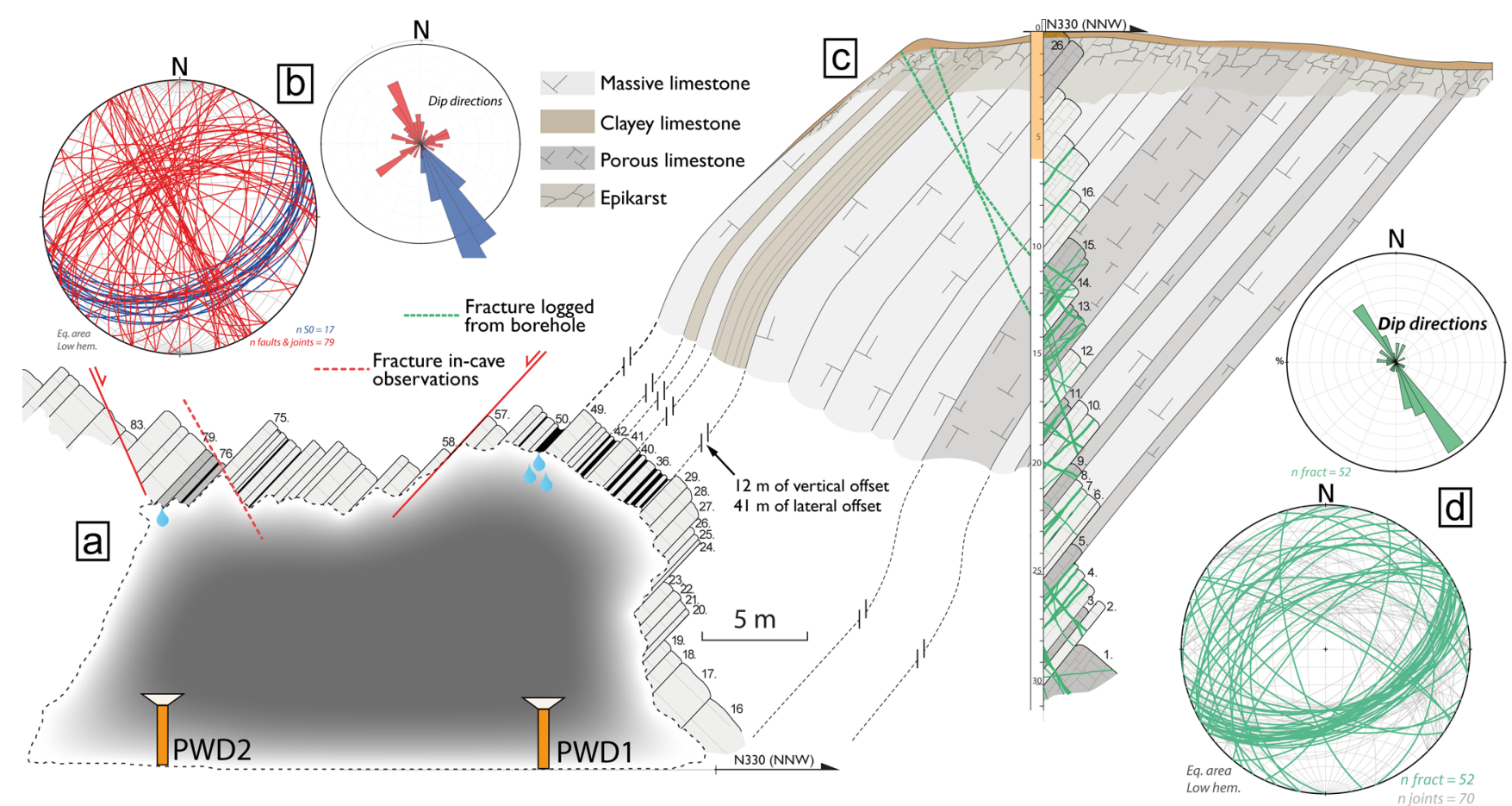

Figure 8. (a) Cross section of the Val d'Enfer room (location highlighted as a dashed line in Fig. 1c), clayey layers (black) and porous limestone layers (dark grey) are highlighted in the massive limestone pile (light grey). Fractures with evidence of displacement are noted in plain red. Two of the percolating water gauges are represented in the cross section (PWD1 and PWD2). PWD3 is located in a smaller passage $40 \mathrm{~m}$ north-west of PWD1. (b) Stereogram (Schmidt lower hemisphere; left) and rose diagram (right) of the planes attributed to bedding planes (blue) and fractures (red), deduced from statistical analysis of a 3-D model of the Val d'Enfer room. (c) Cross section corresponding to the ERT profile (see location in Fig. 1) based on logging of core samples and image survey of the borehole. Main plans are highlighted in green (sedimentary discontinuities or parallel fractures). Dashed green represent the extrapolation of two main fractures towards the surface of the profile. (d) Stereogram (Schmidt lower hemisphere; bottom) and rose diagram (up) of the plans highlighted in green in 2c. Note that (a) and (c) are separated by vertical and lateral offsets of 12 and $41 \mathrm{~m}$ respectively.

tary layers, fractures and joints, and faults) as well as a precise lithostratigraphical log of inaccessible outcrops from the cave's roof (Triantafyllou et al., 2016). The Val d'Enfer room, which starts $20 \mathrm{~m}$ east from the first electrodes of the ERT profile (Fig. 1c), gives a large picture of some of the limestone layers on top of which the ERT profile is attached, in the slope that joins the sinkhole and the cave chamber.

The RCL site was also drilled $2 \mathrm{~m}$ away from the centre of the ERT monitoring location (Fig. 1c), just $35 \mathrm{~m}$ upstream of the northernmost conduit of the RCL. The $31 \mathrm{~m}$ deep borehole was core sampled and surveyed with downhole well logging and imagery. Oriented with respect to the north, it provides complementary information on the lithostratigraphy and structural constraints of the area monitored with ERT.

In the absence of faults with significant offset, the sedimentary layers crossed by the borehole, added to those visible in the Val d'Enfer room, comprise all the geological strata directly sampled by the ERT permanent profile. Although the borehole and field surveys in the cave provide direct observations of the geological bodies, they cannot inform about pos- sible lateral variations in terms of karstification processes and local porosity, which are likely to vary vertically and laterally in such a karst environment. However, field observations suggest that the lithological nature of each layer observed in the borehole or in the cave is expected to remain constant.

\subsection{Structural and lithological context of the Lorette cave}

Figure 8 summarises the structural observations of the Val d'Enfer room (a) and the imaged borehole (c). On the southern side of the Val d'Enfer room, massive limestone layers (46 to 49 in Fig. 8) correspond to the strata on top of which the ERT monitoring profile is installed in the slope of the sinkhole. A succession of thin clayey limestone (layers 31 to 45) is visible just below. This clay-rich layer is associated with higher percolation discharges than in the rest of the room. This pile of clayey limestone can be simplified as two main clayey layers separated by a more massive limestone, as drawn in Fig. 8c. Underlying layers (29 to 16) show a remarkable homogeneity in terms of lithology or weathering rate. They correspond to the first layers cross-cut by the 
borehole, where the first $3.4 \mathrm{~m}$ core samples, compact in the cave outcrop, exhibit higher weathering rates, porosity and fracture intensity, typical of an epikarst layer. Deeper in the borehole, the underlying $7.5 \mathrm{~m}$ (layers 20 to 16) is mostly massive poorly weathered limestone cross-cut by only few joints. The area between 11 and $16 \mathrm{~m}$ deep (layers 15 to 13 ) is characterised by a high number of joints as well as sedimentary discontinuities and beddings partially to totally karstified, which is also evidenced by an increased porosity.

Constraining the geometry of discontinuities (i.e. joints and sedimentary beddings) is crucial to understand the dynamic of the local water infiltration. Some of the encountered open joints are 2 to $3 \mathrm{~cm}$ wide. The openings with strike azimuth N070 and dip values around $50^{\circ}$ mainly represent bedding planes. Based on their geometry, three groups of joint orientations have been identified on the borehole and in the cave (see stereograms and rose diagrams in Fig. 8b-d). The first one comprises planes striking N070 strongly dipping $\left(\sim 50^{\circ}\right)$ to the south. They consist of joints and few faulted structures subparallel to the bedding orientation, similar to the active fault network evidenced by Vandycke and Quinif (2001). Those faults are marked by downdip slickenlines (Fig. 8a) resulting from normal displacement. A second major joint orientation is marked by comparable striking values (N070) but with higher dips $\left(\sim 60^{\circ}\right)$ to the north. In the Val d'Enfer, downdip slickenlines were recognised on these faults showing normal displacement. Normal kinematics are also attested on the borehole log showing displaced sedimentary layers on both parts of few N070-N60 faults. The latter could represent conjugated faults to the direction of the first fault reported above but need additional microstructural surveys. One particular fracture with that orientation is also visible in the northernmost gallery of the Lorette cave and can be followed along $25 \mathrm{~m}$ parallel to the gallery, with an opening of $4-5 \mathrm{~cm}$. A third joints subset shows subvertical joints mainly represented by a mean strike around N150 and few conjugated joints striking N330 (Fig. 8).

In the 2-D geological model of Fig. 8c, two main open fractures with respective orientation N070-N60 and N300NE80 identified in the porous layers (15 to 13) are extrapolated to the surface, as they may play a major role in the water percolation. Figure 8 also highlights the fact that the drip discharge gauges do not directly collect percolating water from the layers below which the electrodes of the ERT profile are installed.

\section{Discussion}

\subsection{Linking resistivity distribution to lithology and microstructures}

As presented in Sect. 4.3, the highest recovered resistivities are around $6000 \Omega \mathrm{m}$, which is a typical signature of lowporosity limestone. Other regions show lower resistivity val- ues that range from 2000-3000 $\Omega \mathrm{m}$ for anomalies 5 and 7 of Fig. 7 to $\sim 500 \Omega \mathrm{m}$ for anomaly 8 . The surface layers ( 1 and 3) also display very low resistivities close to $500 \Omega \mathrm{m}$ on average, which gradually increase with depth up to 1000$2000 \Omega \mathrm{m}$ for the subsurface layer (2). With the karst environment in mind, there are two main explanations for such conductive anomalies: a greater clay vs. limestone ratio or a higher moisture content. On the one hand, a clay-rich composition is already observed in the very thin soil layer. Bedding planes and large open fractures could also be filled with clays or a mixture of clays and percolating water. On the other hand, if conductive anomalies are to be explained with higher moisture contents, they reveal at the same time areas with higher porosity. It is therefore very likely, and hence not surprising, that the ERT profile samples subsurface regions with different degrees of porosity. It remains to be determined whether such increased porosities would account for matrix or conduit porosity types, both frequent in karst subsurface.

The structural model presented in Fig. 8 is an important source of information in order to further investigate the spatial resistivity distribution. It can be summarised as a series of massive limestone strata that includes a pile of clayey limestone layers next to the slope of the sinkhole, porous limestone strata with a greater fracture intensity in the middle and massive limestone layers interbedded with three thin porous limestone strata in the northern part of the ERT profile (Fig. 8). All these strata are dipping $\sim 55^{\circ}$ to the south. Several largely opened fractures are also visible in the borehole image, with two of the most important ones cutting across the inflection point of the profile's topography when extrapolating their $\sim 65^{\circ} \mathrm{N}$ dip. They are representative of one of the preferential fracture directions evidenced in the cave. Finally, the top layer hosts the thin soil layer and a $\sim 3 \mathrm{~m}$ porous, fractured and weathered layer that refers to epikarst features, as highlighted by the core samples.

ERT forward modelling provides a useful tool to verify structural hypotheses, to test their electrical resistivity response and hence to guide the interpretation. In an attempt to account for both the structural information (Fig. 8c) and the ERT results (Fig. 5), the karst underground has been segmented in six resistivity regions (Fig. 9a). The lithological pieces of information are converted into resistivity values using assumptions chosen to best fit the observed data. The fractured area $(<1 \mathrm{~m}$ wide) is given a very conductive value of $200 \Omega \mathrm{m}$ to simulate high clay and moisture contents. A value of $600 \Omega \mathrm{m}$ is given to the clayey limestone layers, while an average value of $3000 \Omega \mathrm{m}$ represents a porous limestone layer. A lower resistivity value is also given to the first limestone layer in the flank of the sinkhole, as a high degree of weathering is observed in that area. The thin soil layer is also represented as an $800 \Omega \mathrm{m}$ layer. These values are chosen to compare with field data acquired in average climate conditions, e.g. not too humid or too dry periods. The epikarst 


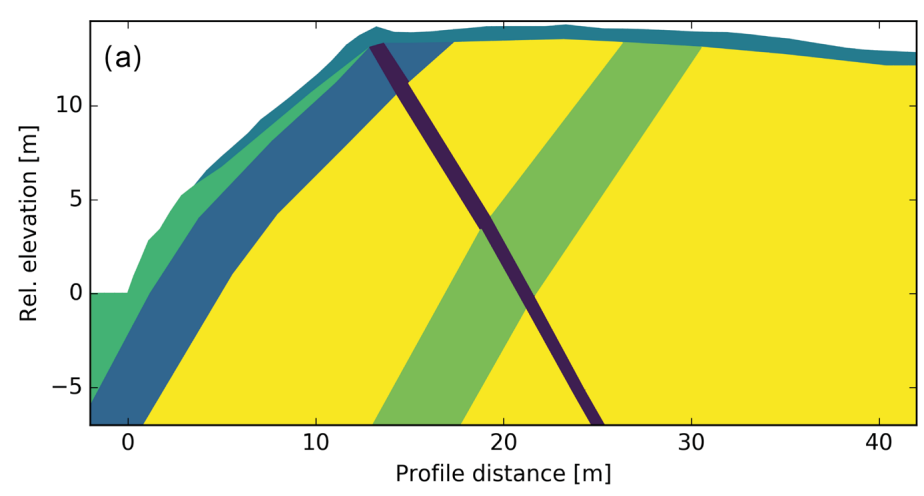

Resistivity model $[\Omega \mathrm{m}]$

200 - Fracture

600 - Clayey limestone

800 - Soil + epikarst

2000 - Limestone doline

3000 - Porous limestone

6000 - Massive limestone

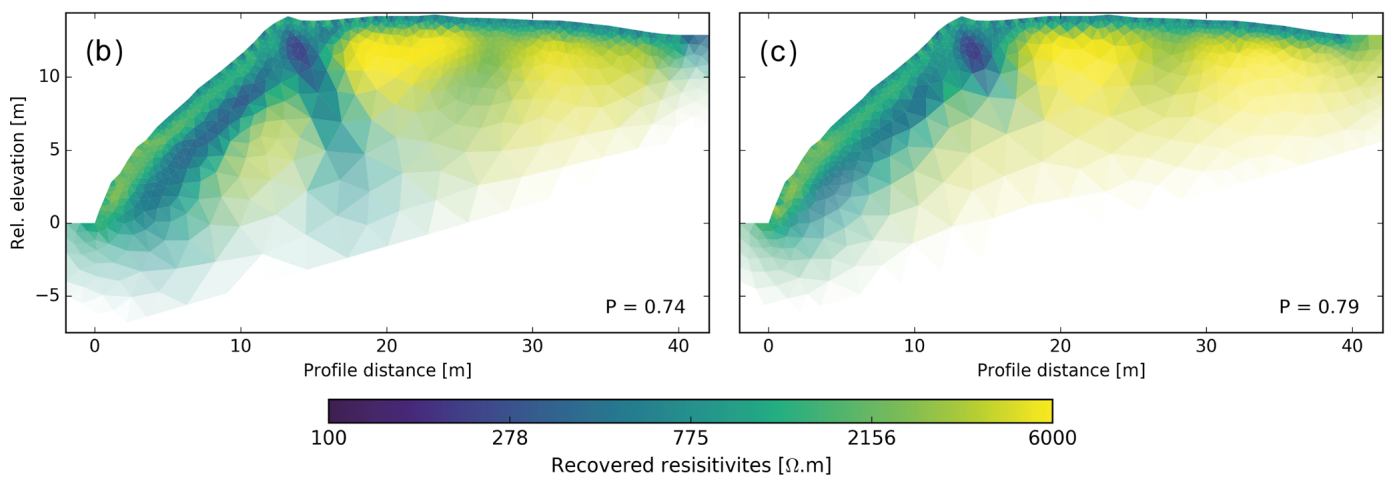

Figure 9. Results of the forward modelling of the resistivity model (a) for the DD protocol (b) and the GD protocol (c). Pearson's correlation coefficients $(P)$ are computed for the data sets of March 2017 presented in Fig. 6.

layer is deliberately omitted on the plateau's side because it is likely to be spatially heterogeneous.

Based on the resistivity model, the potential of each quadrupole of a given protocol is computed by forward modelling, resulting in a synthetic apparent resistivity data set. This synthetic data set can therefore be inverted and the resulting ERT model can be compared to that produced with observed data. Synthetic apparent resistivities are computed using BERT forward model for full DD and GD protocols (i.e. without removing electrode \#12) together with a randomly distributed noise level of $5 \%$. The synthetic data sets are inverted afterwards with an error model defined as the mean error distribution used for the inversion of the whole DD and GD field data time series.

Figure $9 \mathrm{~b}$ and $\mathrm{c}$ present the results of the DD and GD protocols respectively. The first remark is that these reconstructed images look very similar to the inverted field results presented in Fig. 5. The sensitivity of the GD protocol does not allow proper recovery of the fracture anomaly while the DD survey successfully images it. Nonetheless, the thickness and the original resistivity value $(200 \Omega \mathrm{m})$ of this fracture area are not properly recovered, which is most likely due to the fact that the thickness of the anomaly is smaller than the electrode inter-distance and model cells sizes. Moreover, the inversion uses a smoothness constraint in the regularisation (Constable et al., 1987) which tends to blur the boundaries between areas with different resistivity values. Using structural constraints on the ERT inversion could have mitigated such effects. However, as stated in Sect. 4.2.2, we chose not to include such constraints to avoid introducing biases.

More interestingly, with a model value of $600 \Omega \mathrm{m}$, the clayey limestone layer is also clearly imaged after inversion of the synthetic data set. In this case, both the thickness and the resistivity value set in the theoretical model are successfully retrieved, albeit with a progressive resistivity gradient at the boundary with massive limestone layers. To explain the moderate resistive circular anomaly in the middle of the plateau, a value of $3000 \Omega \mathrm{m}$ was chosen for the porous limestone. This leads to a poor reconstruction of this layer, resulting in a $\sim 8 \mathrm{~m}$ deep circular anomaly surrounded by high resistivities accounting for the massive limestone regions. This is both attributed to the lack of resolution at greater depth and the lower contrast in resistivities between the altered and the massive limestones. In any case, such a circular anomaly compares well with that identified in the inversion of field data of the DD surveys and to a lesser extent of the GD surveys. This means that this circular anomaly can successfully be explained as a limestone layer with lower resistivity than surrounding massive limestone strata, for which the low imaging resolution at depth results in averaging the resistivity with that of its surroundings, i.e. the massive limestone. 
Overall, without taking into account an epikarst layer that is thought to explain the small irregular conductive anomalies in the northern side of the inverted field data results, the inversion of these synthetic data sets explains the resistivity distribution substantially. Pearson's correlation coefficients of 0.74 and 0.79 , for the DD and GD surveys respectively, were also computed to compare these synthetic models with inverted models of March 2017 (Fig. 5), representative of average moisture conditions at the RCL site. Such correlation coefficients support the visual similarities between the synthetic and observed images. In the upper layer of the measured data sets, areas enlarging the thin conductive layer simulated in Fig. 9 are therefore interpreted as a signature of epikarst features.

Figure 10 summarises the interpretation of the spatial resistivity distribution made in the light of structural observations and ERT forward modelling. The eight regions of Fig. 7 can now be named as follows: soil plateau (1), epikarst plateau (2), epikarst sinkhole (3), matrix plateau (4), porous limestone (5), massive limestone (6), clayey limestone (7) and fracture (8). Note that the highly conductive anomaly near the bottom of the sinkhole is interpreted as the beginning of a fractured area dipping $30^{\circ}$ to the north, as many of them are evidenced in the borehole logs.

\subsection{Time-lapse ERT to image karst hydrological processes}

In a limestone context such as the RCL site, low resistivities indicate either clay-rich areas or porous areas in humid conditions. However, only the moisture is subject to change on an annual basis. With that in mind, the resistivity variations of the eight regions defined in Fig. 7 can be tracked. The fact that the resistivity values of these regions seem to evolve distinctly regarding climate conditions points to different hydrodynamic behaviours coexisting very close to one another, in agreement with the percolating water discharge data sets. Figure 11 shows the temporal evolution of the median of the absolute resistivity values in the eight regions. GD data sets were chosen for superficial regions $(1,2,3$ in Fig. 7) given the better resolution of that protocol at shallow depths. Resistivity values of the deeper regions $(4,5,6,7,8)$ come from the DD surveys, because of the greater imaging resolution at depth and especially the inability of GD arrays to properly image the fracture anomaly. It results in fewer but better constrained data points. To compensate for the lack of DD surveys, especially during winter 2016, corresponding GD data sets are added with a transparent mask as a guide in Fig. 11. Note that resistivity values of the surface layers from DD surveys and their temporal variations compare well with those of the GD surveys.

The first point to stress is that, as already visible in Fig. 6, all the regions experience a seasonal resistivity variation that seems related to the effective rainfall distribution. The time series displays two annual cycles: April 2015 to March 2017.

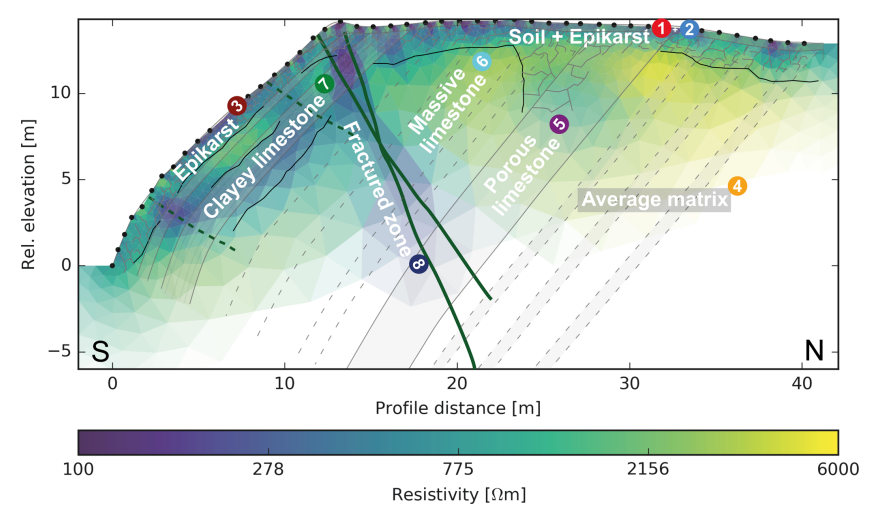

Figure 10. Superposition of the geological model from Fig. 2 on ERT results from March 2017, highlighting the interpretation of the resistivity distribution and anomalies. Numbers of the resistivity clusters defined in Fig. 7 are added for legibility.

Data from 2014 comprise one survey in February, another one in March and a daily series from April to June, which confirm the relatively low resistivity conditions attributed to the end of winter and the beginning of spring. This correlates well with the positive monthly effective rainfalls that are responsible for a general recharge of the soil moisture and groundwater reservoirs, hence resulting in lower resistivity values. A general increase in resistivity is noted when the effective rainfalls become negative in spring, with slight delays in deeper regions. The slope of this increase varies by several orders of magnitude from one region to another - superficial regions showing the sharpest increases, especially on the plateau side of the ERT profile. The massive limestone region displays a seasonal variation with the highest absolute amplitude (note that Fig. 11 displays the log of resistivity), which is associated with the highest average resistivity. The area that shows the lowest seasonal amplitude is the clayey limestone region, with only $220 \Omega \mathrm{m}$ between the minimum in spring and the maximum in winter. The greatest delay with respect to the seasonal dynamic of the surface layers is encountered in clayey limestone region. The porous limestone region on the plateau side exhibits an intermediate behaviour rather close to that of the massive limestone and the sinkhole superficial regions, albeit of greater amplitude.

Lower seasonal amplitudes have two possible causes: higher minimum or lower maximum groundwater contents reached in summer or winter respectively. In similar lithological compositions, if some regions experience lower groundwater deficits, this would result in lower resistivity variations. However, these regions exhibiting such low resistivity variations are also those characterised by low resistivity distribution $(<500 \Omega \mathrm{m})$. This calls for a second hypothesis, i.e. clayenriched layers. According to Waxman and Smits (1968), the greater the clay content, the lower the bulk electrical resistivity amplitude between dry and saturated state. Observations 


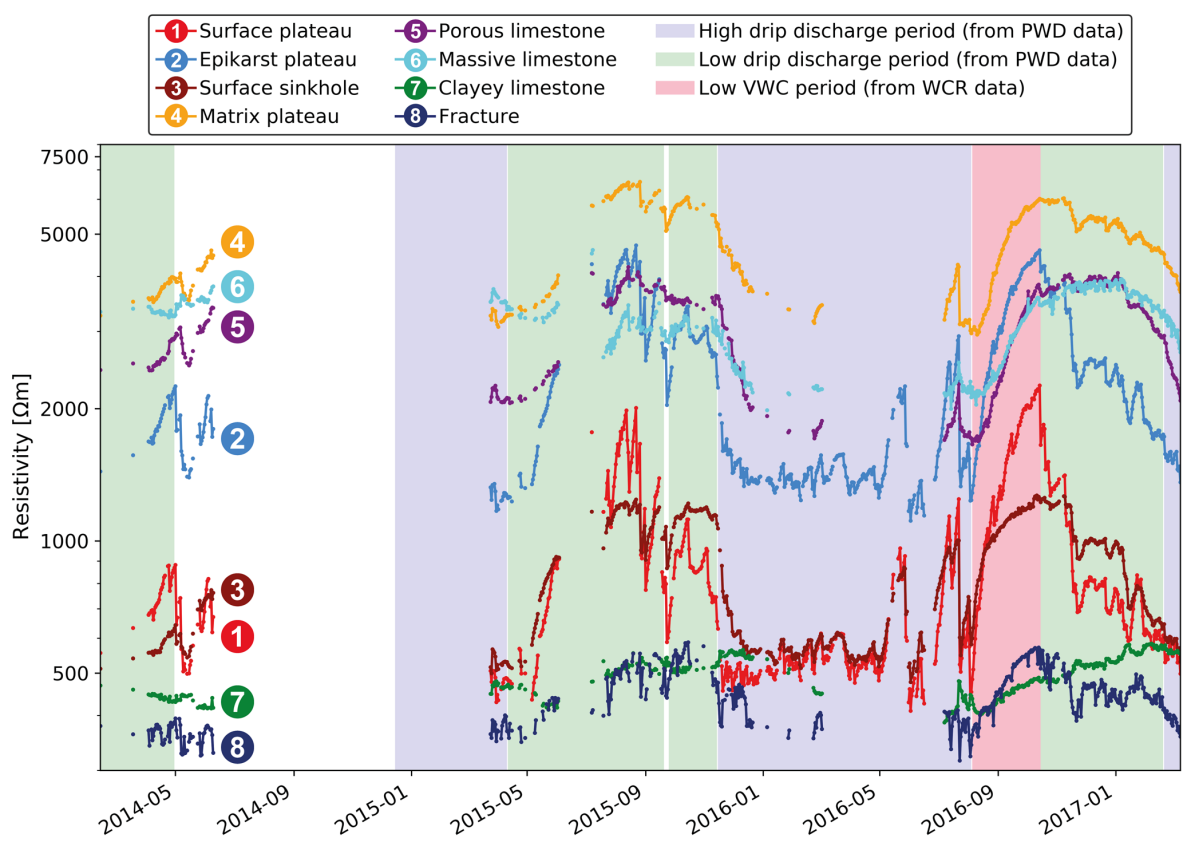

Figure 11. Resistivity time series for the eight regions detailed in Fig. 7, expressed as the median of the log resistivity in each region. The median was chosen, rather than the mean, to limit the contribution of extreme values not representative of the robust central tendency of the cells of each region, especially at their boundaries. The dry and wet periods described in Fig. 3 are also included.

in this area reveal clayey limestone, which supports that second hypothesis.

\subsubsection{Specifying limitations for relating resistivity variations to groundwater content changes}

Converting resistivity variations to groundwater content changes is one particular advantage of ERT monitoring. However, this requires specific site characteristics in terms of subsurface homogeneity that a complex karst system may not offer. Figure 12 presents the co-evolution between data of the $10 \mathrm{~cm}$ deep WCR and the resistivity of the surface layer on the plateau side of the ERT profile. The porosity of the layer was estimated based on the maximum VWC value reached (0.33), which represents the wettest situation measured at a depth of $10 \mathrm{~cm}$. Assuming that this corresponds to a state very close to saturation, this provides a first estimate of the porosity in the top layer. Depending on the rainfall and $\mathrm{ET}_{0}$ conditions, the relationship between saturation and resistivity displays several distinct trends in Fig. 12. Each of the trend lines generally corresponds to a separate drying episode that follows rainfall events, as highlighted by the time colour map in Fig. 12. A particularly long trend line reaches very low saturation values. It corresponds to the August and September 2016 drought. Theoretical relationships derived from Archie's law (Archie, 1942) are also displayed, taking variable pore-water conductivities, as comparative standards. Other relationships requiring more parameters to be fixed (Garré et al., 2011; Revil et al., 1998; Rhoades et al., 1976; Waxman and Smits, 1968) have been tested, hence resulting in similar conclusions: the existence of different resistivity and saturation trends highlights a variable pore-water conductivity. In particular, the trend line of August and September 2016 drought may be attributed to a progressive increase in the pore-water conductivity. Following a rainfall event, the part of the rain water that is not rapidly flushed in the matrix keeps mixing with pore water while increasing ionic leaching. As a result, without significant rainfall events, groundwater resistivity progressively decreases together with an increasing presence of ions in solution. The effect of the important aquatic subterranean fauna usually present in the epikarst layer (Pipan and Culver, 2005; Sket et al., 2004) on the electrical conductivity remains poorly studied. Given the low seasonal amplitude of the electrical conductivity variations measured in the percolating water, it is not thought to play a major role at the RCL site.

As shown in Fig. 2d, the specific conductivity of the in-cave drip discharge shows a quite stable behaviour $(\sim 30 \Omega \mathrm{m})$. Increases in pore-water resistivity after rainfall events, however, reach up to $\sim 65 \Omega \mathrm{m}$, which is attributed to a greater income of fresh rain water directly percolating through fractures. The mixing between rainwater with highly variable conductivity and groundwater may be either progressive through the vadose zone or concentrated in the epikarst. In any case, the significantly lower conductivity of rainwater and the increase in groundwater saturation after rainfall events have opposite impacts on the measured apparent resistivity of the rock. Such a process may therefore 


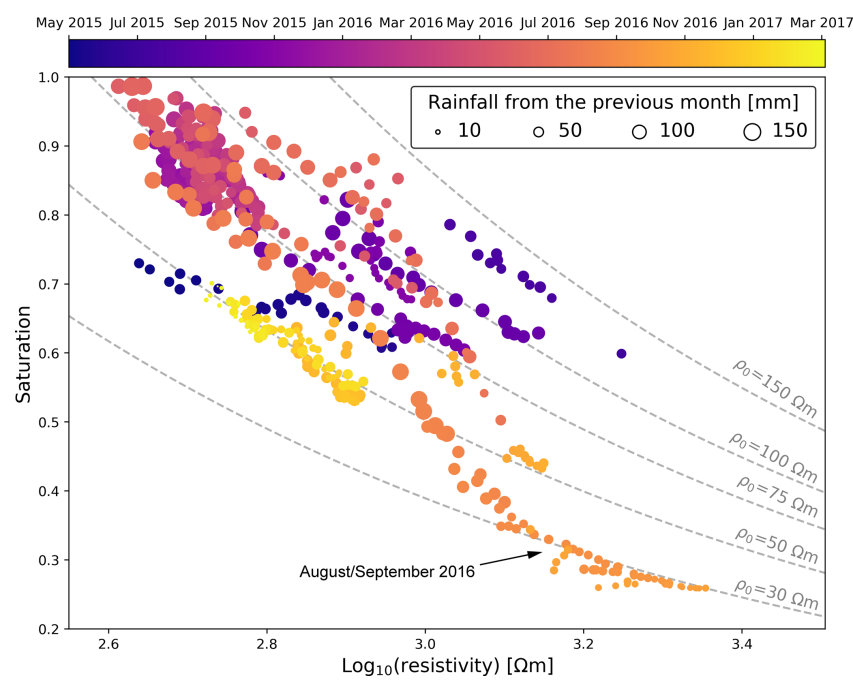

Figure 12. Relationship between the resistivity (median) of the surface limestone region and the saturation from the $10 \mathrm{~cm}$ deep WCR data. Theoretical relationships derived from Archie's law with successive pore-water conductivity values are displayed as watermarks (Archie's cementation and saturation parameters are set to 1.5 and 2 respectively).

tend to underestimate moisture contents deduced from electrical resistivity data after rainfalls. It is responsible at least for a greater uncertainty that enlarges the error on estimated moisture content variation.

Therefore, Fig. 12 illustrates the complexity inherent to the estimation of water content from ERT data even if interesting interpretations may come out of case studies that assume constant pore-water conductivity (e.g. Beff et al., 2013; Brunet et al., 2010; Chambers et al., 2014; Garré et al., 2011; Michot et al., 2003). Such assumption can clearly not be the stated here. Likewise, Uhlemann et al. (2016b) point out similar limitations, attributing abnormal resistivity changes in wetlands to pore-water conductivity variations, which also results in the inapplicability of converting bulk electrical resistivity to moisture contents.

In such a heterogeneous karst context as the RCL site, other important limitations for estimating groundwater contents derived from ERT data concern the porosity, the clay content and the calibration of fitting parameters of petrophysical relationships (Archie, 1942; Revil et al., 1998; Rhoades et al., 1976; Waxman and Smits, 1968). Additionally, absolute electrical resistivity values imaged after inversion, especially for high resistivities, remain dependent on inversion parameters and resistivity contrasts, which mitigate the accuracy of the results. In the absence of precise calibration factors, determining groundwater contents from ERT measurements, even time-lapse, remains highly challenging in a karst environment. Nonetheless, the identification of variable dynamics attributed to groundwater content changes in different spatially limited areas of the subsurface may help in developing hydrological models applied to the vadose zone of complex karst systems.

\subsubsection{Seasonal recharge processes}

To be able to qualitatively analyse the temporal resistivity behaviour of each defined region as a characteristic of their hydrological dynamic, Fig. 13c and d present the median of difference in $\log$ resistivities with respect to dry conditions (15 October 2016), as defined in Eq. (1), for the plateau side and the sinkhole side regions respectively. This allows effective comparison of the resistivity evolution from each region and with environmental data. As pointed out by Samouëlian et al. (2005), looking at changes in resistivity rather than the absolute values eliminates systematic errors. Data from three of the five WCRs are shown in Fig. 13a. Data of the three drip discharge stations are presented in Fig. 13b while positive effective rainfalls are displayed in Fig. 13e.

Effective rainfall and data of the $10 \mathrm{~cm}$ WCR probe are affected by the surface weather conditions as mentioned in Sect. 3. At first sight, three resistivity dynamics can be identified with respect to surface conditions: (D1) regions that correlate the most with the shallow WCR probes, and thus with the fracture drip discharge (PWD1); (D2) regions that exhibit a delay in the increase and the decrease in resistivity, hence correlating more with the deeper WCR probes and especially with the matrix (PWD2) and stalactite (PWD3) drip discharge; and (D3) the fractured region that, despite showing a damped behaviour close to the second group, is characterised by higher variability in response to separate rainfall events.

Superficial regions on the plateau side of the profile, attributed to the soil and epikarst, are very dynamic and hence are members of the D1 type. This is in accordance with Klimchouk (2004), defining the epikarst as a dynamic system. The sharp decrease following rainfall and progressive increases during every period without significant rain testifies to the strong hydrological relationship with the atmosphere, i.e. precipitation and evapotranspiration. The soil and epikarst layers have their moisture contents directly monitored with the WCR probes. A decrease in resistivity is noticed following rainfall events, usually for effective rainfall greater than $2 \mathrm{~mm}$. Such a behaviour is typically observed in soil layers and has been reported in numerous ERT monitoring studies, including karst landscapes (e.g. Brunet et al., 2010; Carriere et al., 2015). Michot et al. (2003) specifically focused on the estimation of soil water content using time-lapse ERT measurements. Seasonally, after a large increase in resistivity through the summer period, recharge of these upper layers is observed and starts in September 2015 and October 2016. A minimum resistivity is reached in December 2015 and March 2017. This seasonal offset is due to the particularly dry fall 2016 and winter 2017. Minimum resistivities should be associated with maximum amounts of groundwater content. 


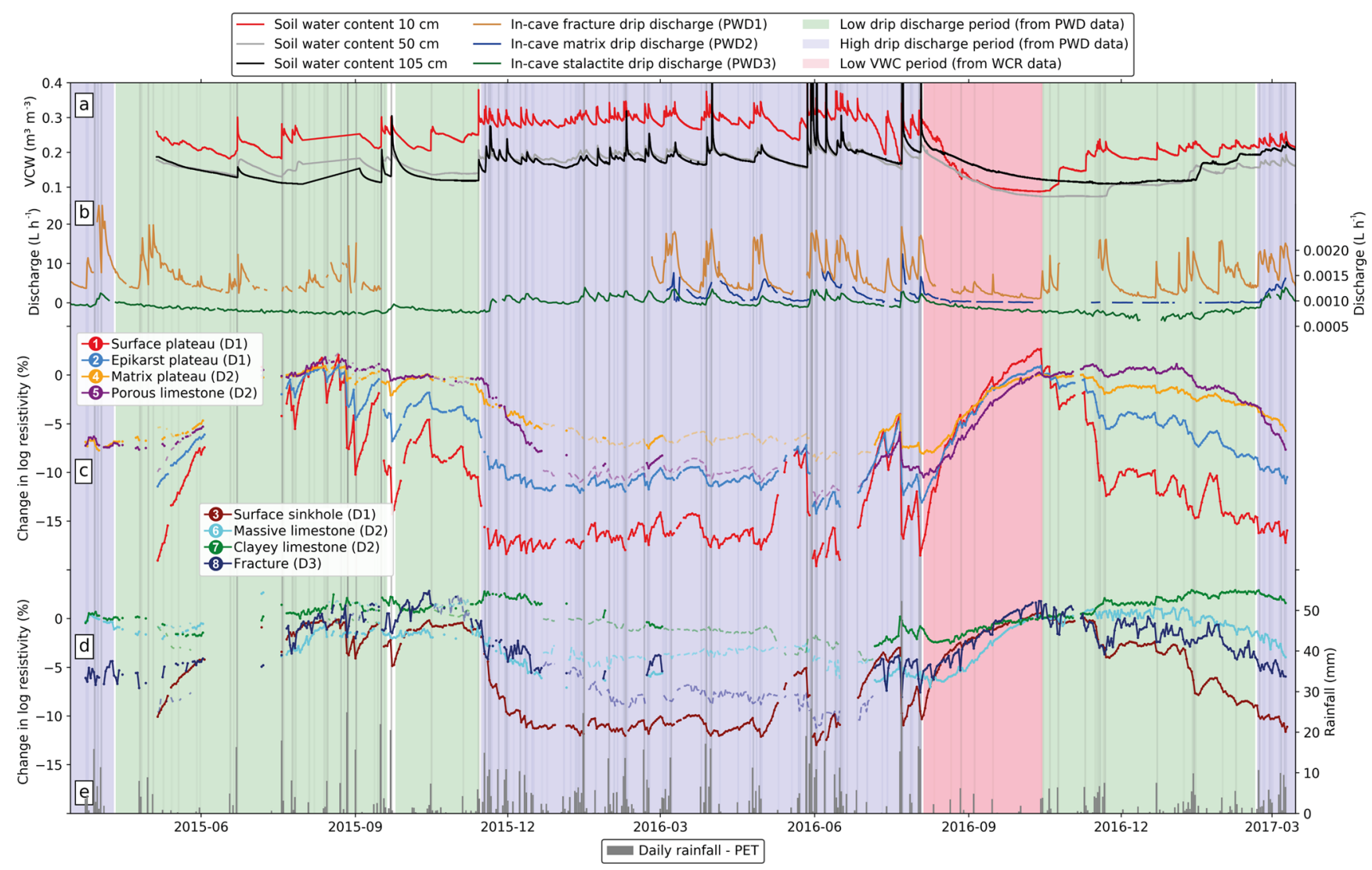

Figure 13. (a) VWC data sets for the 10, 50 and $105 \mathrm{~cm}$ deep WCR probes. (b) Percolating water discharge of the PWD1, PWD2 (left $y$ axis) and PWD3 (right $y$ axis) data sets. (c) and (d) Evolution of changes in log resistivity relative to dry period (15 October 2016) for the eight regions from Fig. 7. (d) Daily effective rainfall. Daily rainfall greater than $3 \mathrm{~mm}$ is also included as spans in the whole figure (transparency as a marker of rainfall intensity) as well as dry and wet periods from Fig. 2.

The relationship between the WCR data and the fracture drip discharge (PWD1), already highlighted in Fig. 2, may therefore be extended to the entire soil layer of the RCL site. The subsurface layer must be characterised by a lower porosity and clay content given its higher average resistivity as evidenced by Fig. 11. However, it shows a dynamic very comparable to that of the surface layer. This highlights the great hydraulic connectivity between the soil layer and the epikarst and hence is typically seen as the principal reservoir that feeds vadose flows (Arbel et al., 2010), in this case the fracture drip discharge station (PWD1). In the presence of a thin soil layer, as it is the case at the RCL site, underlying materials, e.g. the epikarst, must act as a reservoir for a considerable amount of groundwater available for the vegetation (Williams, 2008). However, the fact that the average resistivity progressively increases from the surface to the subsurface layer must also be interpreted in the light of the smoothness constraint of the inversion.

The surface of the slope of the sinkhole also displays a D1type dynamic, albeit more damped. Sharp decreases in resistivity are still observed following major rainfall events, but the seasonal dynamic is less marked, with a less clear cor- relation with the WCR probes. This is most likely due to (i) runoff processes occurring in this zone given the strong topography or (ii) to a very poor capacity in terms of water retention because of the progressively thinner soil layer. The fact that no specific resistivity variations are noticed in Fig. 6, next to the first five electrodes at the bottom of the sinkhole, is in agreement with the absence of soil layer in that area and the subvertical topography where surface runoff is likely to be predominant. This justified why this area was not included in the surface region tracked in the slope of the sinkhole as displayed in Figs. 7, 11 and 13.

D2 dynamics are attributed to the deeper regions comprising the clayey limestone, porous limestone, massive limestone and the matrix to the north of the plateau. The three latter regions exhibit a very similar seasonal variability - the massive and porous limestone regions reaching their maximum approximately at the same time, $\sim 25$ days after the D1 types (Fig. 13c-d). The matrix on the plateau side shows a slight negative offset, reaching its maximum closer to that of the D1 types. These D2-type regions stay in high resistivity conditions for approximately 75 more days: until the end of November 2015 and the end of January 2017, fol- 
lowing a particularly dry fall and winter. Conversely, the clayey limestone, despite a much lower seasonal amplitude, reaches its maximum resistivity more than 4 months after the D1 types. Overall, the similarity between the D2 curves and PWD2 or PWD3 data sets is indubitable. As shown in Fig. 13, the top of the D2 curves corresponds to dry periods identified from drip discharge data, except for the clayey limestone for which it corresponds only to resistivity increases. The reactivation of groundwater recharge in this layer, evidenced by a general decrease in resistivity in winter, can be correlated to the reactivation of PWD2 and PWD3 high drip discharge regime. For the other D2-type regions, the curve starts decreasing when the slope of the PWD3 curve turns positive in dry periods, i.e. in December 2015 and January 2017 (Fig. 13c-d).

A delay is also noticeable at the beginning of the resistivity increase, especially during the 2016 dry period, in the porous limestone and more strongly in the massive and clayey limestone curves. This lag period reaches 15 days in August 2016. Such trends attest that these deeper regions stay less affected by the surface conditions in the beginning of drying processes. This indicates delayed infiltration in deeper areas. Then the rise in resistivity is significant only after the upper layers reach $\sim 75 \%$ of their increases - the surface of the plateau side having already risen from -20 to $-5 \%$ change in log resistivity. Similarly, the decrease in the D2 curves takes place mostly during winter, when the upper layers are already lowered close to their minimum value.

The fractured region also exhibits a dynamic close to the D2 type, yet on which a greater variability in response to rainfall events is superimposed. Such resistivity decreases are different from the response of surface layers that typically shows a sharp decrease depending on rainfall intensity followed by a slower increase, which corresponds to soil drying processes evidenced by the WCR data. In the fractured region, the resistivity perturbation induced by rainfalls is more ephemeral, with the resistivity curves retrieving most of the time the resistivity value prior to precipitation 1 day after the rainfall event, which mimics the sharp recession curves following rainfall events displayed by PWD1 data.

\subsubsection{Resistivity response to rainfall events}

Figure 14 particularly focuses on ERT data on the rainfall event scale, showing cross-correlation functions of the eight resistivity regions with the environmental variables on a lag window of -5 to +5 days. In order to consistently correlate these different types of data sets, the first derivative of the resistivity time series was selected and cross-correlated with the rainfall, the first derivative of the 10 and $105 \mathrm{~cm}$ WCR data, and the first derivative of the log PWD data sets. The first derivative highlights the variations of the measured variables from one day to the next, while centring the time series on 0 . Taking the log of the PWD data sets provides a way to account for minor variations in dry periods. To take into ac- count the different sampling rates of all the data sets, cumulative rainfalls and mean WCR and PWD values are computed within $8 \mathrm{~h}$ windows from $6 \mathrm{~h}$ prior to $2 \mathrm{~h}$ after each ERT time step. Such time windows account for the average delay necessary for a rainfall event to be detected in the $10 \mathrm{~cm}$ WCR probe and PWD1 data, which are the monitored spots that are most reactive to infiltration of rain water.

As a result, most of the correlation peaks occur at lag 0 or lag 1 day. No significant peaks are noticed at negative lags, except for the clayey limestone region that exhibits a behaviour totally different from the rest. This will be discussed further in this section. For other regions, no interpretation can therefore be drawn concerning variations of resistivity that could precede variations of the environmental variables. D1-type regions exhibit very similar patterns, being unsurprisingly most negatively correlated either to the rainfall, the $10 \mathrm{~cm}$ WCR or the PWD1 data sets at lag 0 day (Fig. 14a, $\mathrm{b}$ and d). This means that decreases in resistivity are mostly concurrent, with respect to the temporal resolution of 1 day, to rainfall events as well as increases in soil moisture content or drip discharge (see Fig. 13). Logically, the crosscorrelation function rapidly decreases with positive lags for the correlation with rainfall and the $10 \mathrm{~cm}$ deep WCR data sets. Resistivity data of the D1-type regions also exhibit nonlagged cross-correlation peaks, although smaller, with the $105 \mathrm{~cm}$ WCR and PWD2 data (Fig. 14c-e). No correlation comes out for any of the regions with PWD3 data (Fig. 14f), which highlights their non-reactivity to storms during dry periods, as visible in Fig. 2. Interestingly, the fractured region exhibits cross-correlation functions similar to those of D1type regions, albeit more damped, displaying peaks with negative cross-correlation coefficients at lag 0 day with all the variables except the PWD3 data. However, the fractured region becomes positively correlated as early as lag 1 or 2 day, suggesting a rapid resistivity increase following a first decrease concurrent to a rainfall event or VWC increases. That particular trend of the fractured region is also visible for the cross-correlation with the PWD1 and PWD2 data sets.

In contrast to this, D2-type regions exhibit patterns different from each other and from those of D1-type regions. The matrix plateau and porous limestone region evolve quite similarly with a cross-correlation peak at 1 day lag for the rainfall, $10 \mathrm{~cm}$ WCR probe and PWD1 data sets, with the matrix plateau region still showing a noticeable cross-correlation coefficient at lag 0 day. This particular region is characterised by a succession of thin massive and porous limestone. The greater number of bedding planes could therefore provide more preferential pathways favouring rapid percolation of rainwater, compared to the porous limestone region. Both regions are correlated without any lag, with the deepest WCR probe and PWD2 data at lag 0 day, similarly with D1-type regions, with the matrix plateau region being even the most correlated with these variables. This confirms on a short timescale the greater contribution of these D2-type regions to the PWD2 seasonal drip discharge. 


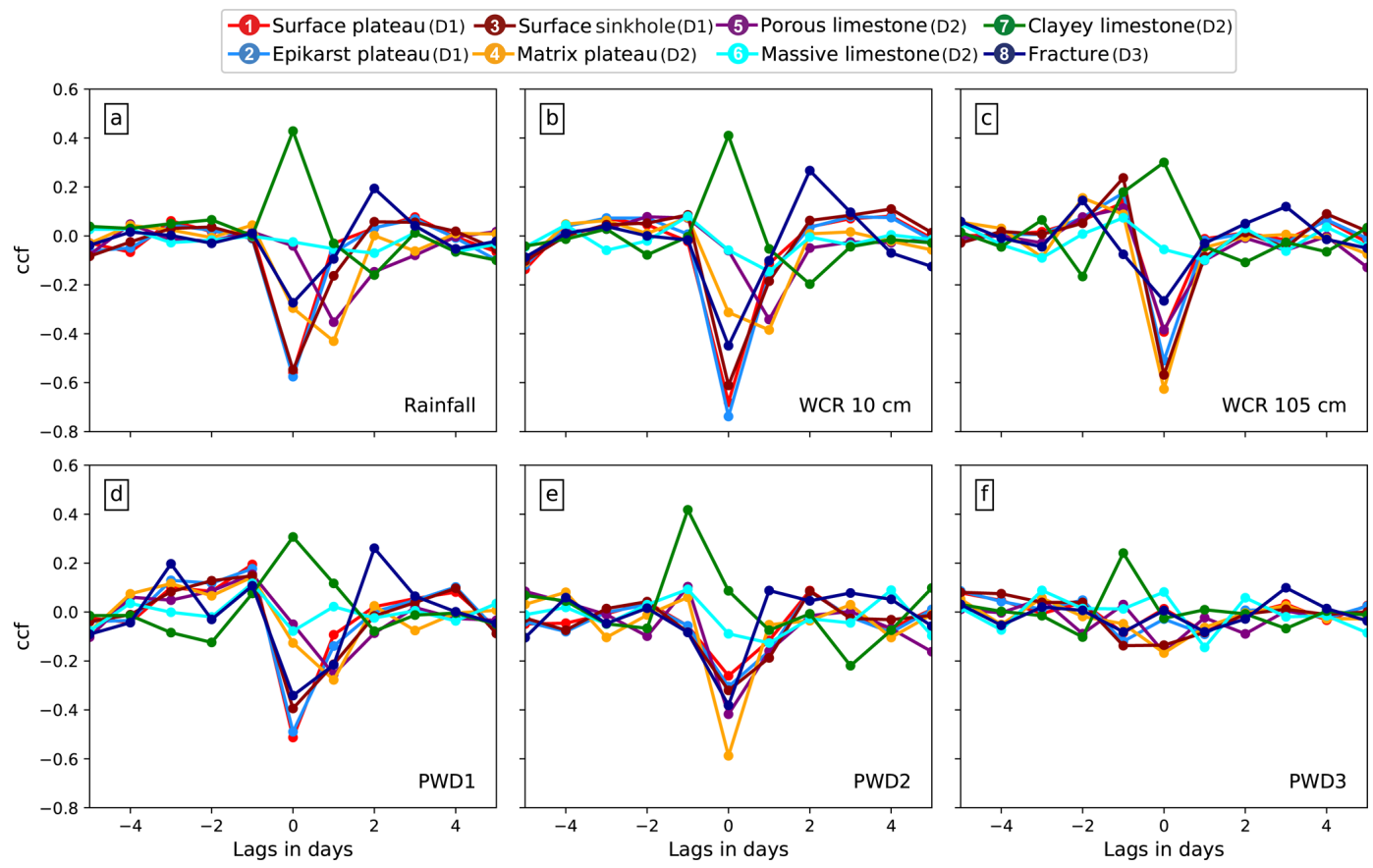

Figure 14. Cross-correlation of the ERT data sets of the eight regions defined in Fig. 7 and environmental data sets.

The porous limestone and rainfall cross-correlation coefficient decreases progressively, being still negatively correlated at positive lag 2 and 3 days. The resistivity of the porous limestone still decreasing 2 to 3 days after a rainfall event is a possible explanation, highlighting a likely diffusion of the rainwater infiltration within the porous limestone layer. Despite being associated with the $\mathrm{D} 2$-type regions on a seasonal basis, the massive limestone does not show any clear correlation with any of the environmental data on the rainfall event scale. This is likely due to the very low to zero rainwater contribution to the bulk resistivity of that area given its low porosity and fracture density.

Finally, the clayey limestone shows a quite surprising trend, being positively correlated at lag 0 day with most of the environmental data. In other words, increases in resistivity are concurrent with rainfall events. This is also particularly visible in Fig. 6b-5 and b-10. We propose two possible explanations of this observation: (i) a time-lapse ERT inversion artefact and (ii) an influx of rainwater more resistive than the actual pore water.

i. The first hypothesis pointing out time-lapse ERT inversion artefacts was already addressed in the case of shallow infiltration monitoring by Clément et al. (2009). They demonstrated using synthetic models that infiltration processes at shallow depths usually produce a decrease in resistivity in the upper layer, while an increase in resistivity may be observed at intermediate depths, where the resistivity is actually not changing. Descloitres et al. (2008) identified such time-lapse artefacts when tracking seasonal recharge processes by
ERT, especially located in the subsurface of slopes. These studies point out that infiltration through a succession of layers with different resistivity signatures may enhance such artefacts. Hence, the relatively complex resistivity distribution in the subsurface of the slope of the sinkhole may favour such artefacts, as the conductive clayey limestone lies below a more resistive layer, which is itself below the surface conductive layer. According to Clément et al. (2009), a proposed solution to avoid such artefacts involves adding decoupling constraints in the inversion along a shallow line evolving together with the infiltration front, determined by external data. This seems in our case quite unrealistic given that the evolution of the infiltration front remains unknown in the clayey limestone. The plateau is likely to provide a major part of the water infiltrating the clayey limestone, given the strong runoff processes expected in the slope of the sinkhole. Hence, a minor part of infiltrating water is still expected to come from the sinkhole itself, especially through open fractures. The hydraulic conductivity is also constrained by the lithological nature of each layer, which complicates the problem. Therefore, the complex infiltration in the clayey limestone justifies not adding such a decoupling line in the inversion. Alternatively, artefacts could also result from the underestimation of the noise (LaBrecque et al., 1996b) when weighting the observed data of each data set for the inversion. The use of reciprocal errors for the DD surveys (used for tracing changes in the clayey limestone in Figs. 11, 13 and 14) must mitigate this possibility. 
ii. The latter proposed hypothesis regarding these increases in resistivity following rainfall events in clayey limestone must also be discussed. In some cases, an influx of a great quantity of fresh, hence less conductive, water into a partially wet clayey material can result in an increase in resistivity, especially given the inverse power relationship between the bulk electrical resistivity and the saturation (see Fig. 12), as evidenced in the model proposed by Waxman and Smits (1968). The only option being that the clayey limestone stays constantly at a high saturation rate, with highly conductive pore water $(\sim 30 \Omega \mathrm{m}$ as shown by the drip discharge electrical conductivity data). An influx of more resistive percolating rainwater $(>100 \Omega \mathrm{m})$ could therefore result in a slight increase in resistivity, even if the saturation rate also increases. While this could occasionally happen following precipitation with particularly low conductivity rainwater, this is unlikely to be predominant in the signal.

\subsection{Resistivity dynamics as markers for karst hydrology}

Overall, the observations concerning the resistivity dynamics of each region are determinant, as they provide an image of the sources of the drip discharge type measured in the cave system. In particular, the nature of each region and its variability in terms of resistivity must be interpreted in conjunction with its likely contribution to the karst hydrodynamic at the RCL site. Firstly, the role of the soil and epikarst is evidenced as being very dynamic (D1) with regard to the atmospheric inputs. This reactivity indicates a limited buffering role for the epikarst in terms of hydrological recharge, as evidenced by previous studies (Aquilina et al., 2003; Bakalowicz, 2005; Ford and Williams, 2008; Genty and Deflandre, 1998; Poulain et al., 2015b; Sheffer et al., 2011). Indeed, the likely very thin epikarst in the middle of the plateau as pointed out by the resistivity images and other field observations strongly limits water storage. Based on the resistivity variations, the epikarst, thicker to the north of the plateau, acts anyway as a buffer during spring, being still a likely source of diffuse infiltration to deeper areas while responding to the water demands of the vegetation. Storms cause ephemeral recharge in these surface layers but also increase rapid infiltration to deeper areas as revealed by the dynamics of the fractured area following rainfall events, even in summer. This second effect seems damped during the summer.

Diffuse flows propagating through the rock matrix are also evidenced with D2 dynamics, which are consistent with previous findings (e.g. Lange et al., 2010; Perrin et al., 2003). Carrière et al. (2016) particularly highlighted via ERT monitoring of rainfall events that considerable amounts of water can be temporarily stored in the vadose zone of karst systems and more specifically in the porous matrix. At the RCL site, the fact that all regions reach a minimum thresh- old in winter periods is also interesting, meaning that they approach their maximum water retention capacity. The relatively weaker effects of winter rainfalls on the resistivity variations compared to those of summer storms testifies to the high saturation present in the rock matrix during such periods. Given the inverse power relationship between saturation and bulk electrical resistivity (Archie, 1942; Revil et al., 1998; Rhoades et al., 1976; Waxman and Smits, 1968) as illustrated by Fig. 12, progressive increases in saturation are expected to result in lower and lower resistivity decreases, as for the drying period of August and September 2016. This means that in relatively porous rock, such as the porous limestone at the RCL site, an important recharge occurs in winter. Due to the aforementioned limitations in accurately estimating groundwater contents, the intensity of the deficit, when the maximum of resistivity changes is reached, remains unconstrained.

The role of the conduit porosity is also clearly evidenced by the greater variability in the fractured region, compared to that of the massive limestone and porous limestone. D3 dynamics can be interpreted as an evidence of the direct hydraulic connectivity between surface layers and post-storm drip discharge of the percolating water (Arbel et al., 2010) as monitored in-cave. Furthermore, the seasonal variation revealed in the fractured zone is an indicator of the likely water exchange from the conduits to the matrix porosity in that area. Enhanced porosity near fracture walls as seen in core samples can temporarily increase stored groundwater. When the fractures are saturated with percolating water after rainfall events, a gradient towards these porous areas may occur, as already modelled in previous studies (Bailly-Comte et al., 2010; Hartmann et al., 2013). Similarly, an input of water from porous areas close to the fracture walls or from crosscut porous layers is likely to occur in dry periods, participating to the vadose baseflow observed in PWD1, as Poulain et al. (2018) found at the RCL site. Alternatively, a slower infiltration can also occur in narrower fractures or in bottlenecks towards the main fractures. Figure 15 summarises these interpretations and the hydrological functions of each of the regions imaged by the ERT monitoring at RCL site.

\section{Conclusions}

This first long-term permanent ERT monitoring of a complex karst vadose zone has revealed seasonal recharge processes with variable dynamics. ERT allowed clustering of distinct areas showing contrasting evolutions through three hydrological cycles. Such different behaviours are attributed to distinct categories of vadose reservoirs responsible for specific percolation types. They could be associated with the sources of distinct percolation drip discharge measured in-cave. This study was able to differentiate three groups with distinct resistivity variations and to link them to sources of in-cave drip discharge: 


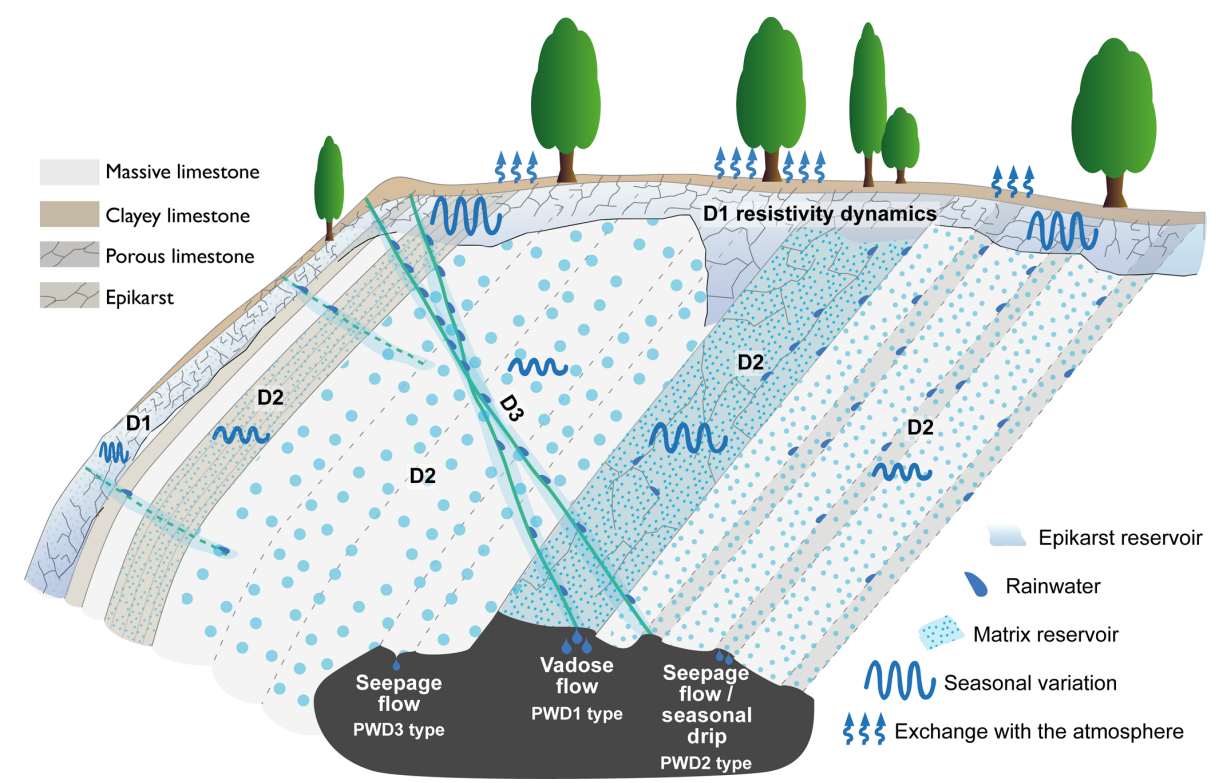

Figure 15. Schematic view of the hydrological processes occurring in the subsurface of RCL site.

- Upper conductive layers, comprising the soil and the epikarst, which are in direct contact with the atmosphere, hence showing the highest variability (D1).

- Deeper regions characterised by a more diffuse and damped seasonal variation, showing a delayed recharge (D2). The resistivity values of these areas depend on the lithology, the porosity and the clay content, which also determine their groundwater retention capacity.

- A conductive fractured zone (D3) exhibiting a dynamic close to that of D2 group, but with a greater variability in response to rainfall events, revealing a preferential pathway for rainwater. Water exchange between conduits and porous matrix is likely to explain the seasonal variation.

These observations are consistent with previous knowledge of hydrological processes in the karst vadose zone (Ford and Williams, 2008; Goldscheider and Drew, 2007), while bringing a first detailed view of the sources responsible for the duality of flows typically observed in karst environments: quick flows and diffuse flows (White, 2002). Moreover, given the small resistivity variations measured in winter, the recharge processes in all areas of the monitored site are expected to be highly efficient. The main constraint on the amount of groundwater volumes stored in the vadose zone appears to be the matrix porosity.

Hydrogeophysical experiments in karst systems, especially targeting the vadose zone, are very challenging, as already raised by Chalikakis et al. (2011). This study proves that, combined with a detailed structural and lithological survey on a local scale, as well as with additional environmental measurements, ERT monitoring is able to image and track through time recharge processes within the vadose zone of a karst system.

Improved ERT inversion strategies for highly heterogeneous environments could provide more constrained results reducing the occurrence of artefacts similar to those experienced in the clayey limestone of our field site. Synthetic modelling approaches could also help in validating the assumptions made on the water infiltration processes. In parallel, 3-D imaging would improve spatial resolution, hence reducing uncertainties as compared to 2-D imaging. In such case, a real effort should be made for optimising more complex measuring protocols with regard to highly resistive karst environments. Clustering tools could also improve the detection of structures in the ERT images, as proposed by $\mathrm{Xu}$ et al. (2017). In such cases, fully taking advantage of the time-lapse information, i.e. distinct resistivity dynamics, seems the most important but challenging aspect.

Combining these techniques with other geophysical methods would also be definitely interesting, e.g. to image hydrological characteristics of the subsurface on larger scales. This is especially the case of passive seismic noise monitoring networks that have recently proved their applicability to track groundwater content variations at several depths (Lecocq et al., 2017; Voisin et al., 2016), even in karst (Fores, 2016).

Overall, these findings support existing models and bring new opportunities to understand the karst system, often modelled as black boxes. Imaging the sources of drip discharge signals conventionally monitored in numerous cave systems contributes to improve the understanding of karst subsurface hydrodynamic behaviours. In particular, the joined analysis between time-lapse ERT results and percolating water mea- 
surements is definitely a novel, promising approach to investigate the sources of distinct in-cave flow types and their lithological and structural constraints. More specifically, this study calls for similar geophysical monitoring to be tested in different karst environments, in combination with conventional hydrological monitoring or dense monitoring networks of cave drip water (Mahmud et al., 2016, 2018), to gather new types of hydrological data to be included in karst hydrological modelling, such as lumped karst modelling of vadose zone infiltration processes. Such novel approaches are required to face future challenges for the management of karst groundwater resources worldwide and the increasing risks of contamination issues raised by the increasing agricultural demands. Better constraining recharge processes of karst aquifers also brings grist to the mill of the study of speleothems, with implications on paleoclimatic researches.

Data availability. Data used in this research paper (comprising ERT, cave drip discharge, soil moisture, rainfall and water conductivity) are available at https://doi.org/10.5281/zenodo.1158631 (Watlet et al., 2018a). ET $_{0}$ data are available via the Pameseb organisation (http://pameseb.be). 


\section{Appendix A}
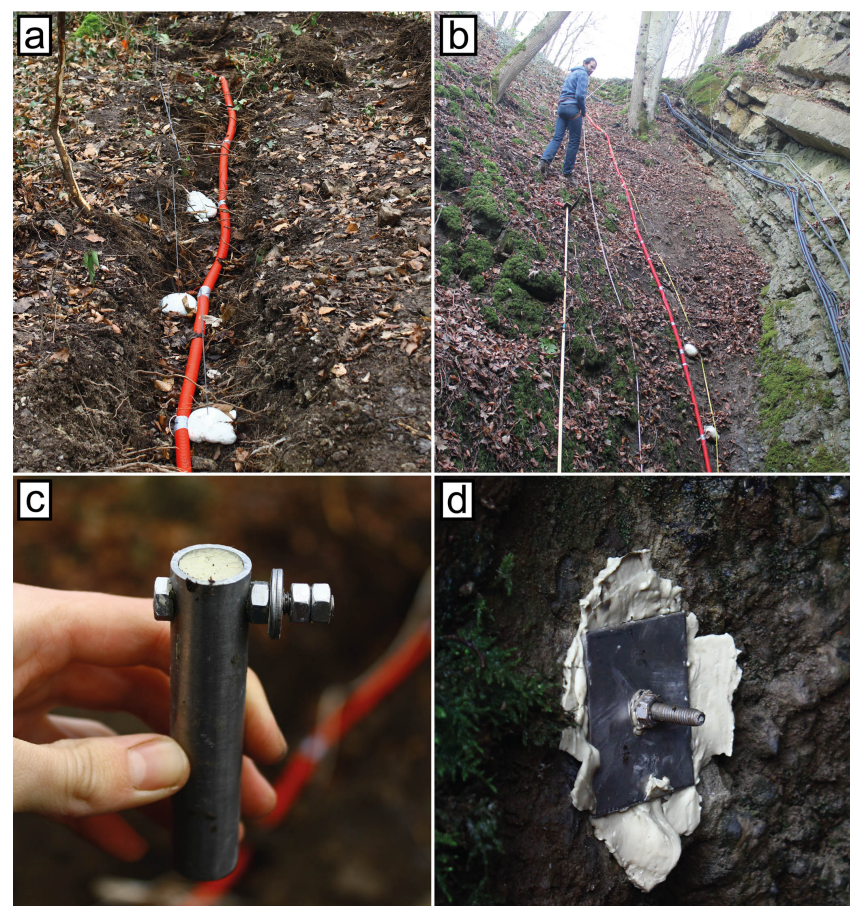

Figure A1. Photos of the ERT profile, with the trench at the top of the plateau (a); the situation in the slope of the sinkhole (b); the stainless steel hollow tubes used as electrodes in most of the profile (c); and the stainless steel plates bolted to the rock at the bottom of the sinkhole (d). 


\section{The Supplement related to this article is available online at https://doi.org/10.5194/hess-22-1563-2018- supplement.}

Competing interests. The authors declare that they have no conflict of interest.

Acknowledgements. This work is part of the Karst Aquifer Research by Geophysics (KARAG) project (karag.be) funded by the Fonds de la Recherche Scientifique (FNRS-FRS). Arnaud Watlet is also supported by the Ernest du Bois Fund, managed by the King Baudouin Foundation. We would like to thank all the colleagues who were involved in this project and particularly Claudio Barcella for field and laboratory works, Christophe Bastin, Bérénice Deletter, Fadel Cissé, Stefaan Castelein, Marc Hendrickx, Giovanni Rapagnani, Sebastian Uhlemann, Fabian Wagner, Thomas Lecocq, Koen Van Noten and Thierry Camelbeeck, as well as the ASBL Grottes de Lorette, Guy Evrard and the team members, and the municipality of Rochefort for their hospitality and support. This paper benefited from Matplotlib (Hunter, 2007), Pandas (McKinney, 2011), Glue (available at http://glueviz.org) and GDAL/OGR packages (available at http://gdal.org) as well as QGis (available at http://qgis.org) software. We also thank one anonymous reviewer and Ty P. Ferre for their thoughtful comments during the open discussion.

Edited by: Ty P. A. Ferre

Reviewed by: one anonymous referee

\section{References}

Allen, R. G., Pereira, S. L., Raes, D., and Smith, M.: Crop evapotranspiration-Guidelines for computing crop water requirements-FAO Irrigation and drainage paper 56, FAO, Rome, Italy, 300, D05109, 1998.

Aquilina, L., Ladouche, B., Doerfliger, N., and Bakalowicz, M.: Deep water circulation, residence time, and chemistry in a karst complex, Ground Water, 41, 790-805, 2003.

Arbel, Y., Greenbaum, N., Lange, J., and Inbar, M.: Infiltration processes and flow rates in developed karst vadose zone using tracers in cave drips, Earth Surf. Proc. Land., 35, 1682-1693, https://doi.org/10.1002/esp.2010, 2010.

Archie, G. E.: The electrical resistivity $\log$ as an aid in determining some reservoir characteristics, Trans. AIME, 146, 54-62, 1942.

Atkinson, T. C.: Diffuse flow and conduit flow in limestone terrain in the Mendip Hills, Somerset (Great Britain), J. Hydrol., 35, $93-$ 110, https://doi.org/10.1016/0022-1694(77)90079-8, 1977.

Bailly-Comte, V., Martin, J. B., Jourde, H., Screaton, E. J., Pistre, S., and Langston, A.: Water exchange and pressure transfer between conduits and matrix and their influence on hydrodynamics of two karst aquifers with sinking streams, J. Hydrol., 386, 55-66, https://doi.org/10.1016/j.jhydrol.2010.03.005, 2010.

Bakalowicz, M.: Karst groundwater: a challenge for new resources, Hydrogeol. J., 13, 148-160, https://doi.org/10.1007/s10040-0040402-9, 2005.
Baker, A., Barnes, W. L., and Smart, P. L.: Variations in the discharge and organic matter content of stalagmite drip waters in Lower Cave, Bristol, Hydrol. Process., 11, 1541-1555, 1997.

Barchy, L., Dejonghe, L., and Marion, J.-M.: Geological map of Wallonia (Belgium), scale 1/25 000, sheet Rochefort - Nassogne no. 59/3-4, Public Service of Wallonia, Namur, Belgium, 2014.

Beff, L., Günther, T., Vandoorne, B., Couvreur, V., and Javaux, M.: Three-dimensional monitoring of soil water content in a maize field using Electrical Resistivity Tomography, Hydrol. Earth Syst. Sci., 17, 595-609, https://doi.org/10.5194/hess-17595-2013, 2013.

Binley, A., Hubbard, S. S., Huisman, J. A., Revil, A., Robinson, D. A., Singha, K., and Slater, L. D.: The emergence of hydrogeophysics for improved understanding of subsurface processes over multiple scales: the Emergence of Hydrogeophysics, Water Resour. Res., 51, 3837-3866, https://doi.org/10.1002/2015WR017016, 2015.

Brunet, P., Clément, R., and Bouvier, C.: Monitoring soil water content and deficit using Electrical Resistivity Tomography (ERT) a case study in the Cevennes area, France, J. Hydrol., 380, 146153, https://doi.org/10.1016/j.jhydrol.2009.10.032, 2010.

Camelbeeck, T., van Ruymbeke, M., Quinif, Y., Vandycke, S., de Kerchove, E., and Ping, Z.: Observation and interpretation of fault activity in the Rochefort cave (Belgium), Tectonophysics, 581, 48-61, https://doi.org/10.1016/j.tecto.2011.09.027, 2011.

Carrière, S. D., Chalikakis, K., Sénéchal, G., Danquigny, C., and Emblanch, C.: Combining electrical resistivity tomography and ground penetrating radar to study geological structuring of karst Unsaturated Zone, J. Appl. Geophys., 94, 31-41, https://doi.org/10.1016/j.jappgeo.2013.03.014, 2013.

Carrière, S. D., Chalikakis, K., Danquigny, C., Clement, R., and Emblanch, C.: Feasibility and limits of electrical resistivity tomography to monitor water infiltration through karst medium during a rainy event, in Hydrogeological and Environmental Investigations in Karst Systems, vol. 1, Springer, Berlin, Germany, 45-55, 2015.

Carrière, S. D., Chalikakis, K., Danquigny, C., Davi, H., Mazzilli, N., Ollivier, C., and Emblanch, C.: The role of porous matrix in water flow regulation within a karst unsaturated zone: an integrated hydrogeophysical approach, Hydrogeol. J., 24, 19051918, https://doi.org/10.1007/s10040-016-1425-8, 2016.

Caterina, D., Flores-Orozco, A., and Nguyen, F.: Long-term ERT monitoring of biogeochemical changes of an aged hydrocarbon contamination, J. Contam. Hydrol., 201, 19-29, https://doi.org/10.1016/j.jconhyd.2017.04.003, 2017.

Chalikakis, K., Plagnes, V., Guerin, R., Valois, R., and Bosch, F. P.: Contribution of geophysical methods to karstsystem exploration: an overview, Hydrogeol. J., 19, 1169-1180, https://doi.org/10.1007/s10040-011-0746-x, 2011.

Chambers, J., Meldrum, P., Gunn, D., Wilkinson, P., Merritt, A., Murphy, W., West, J., Kuras, O., Haslam, E., Hobbs, P., Pennington, C., and Munro, C.: Geophysical-Geotechnical Sensor Networks for Landslide Monitoring, in: Landslide Science and Practice, Springer, Berlin, Heidelberg, Germany, 289-294, 2013.

Chambers, J. E., Gunn, D. A., Wilkinson, P. B., Meldrum, P. I., Haslam, E., Holyoake, S., Kirkham, M., Kuras, O., Merritt, A., and Wragg, J.: 4-D electrical resistivity tomography monitoring of soil moisture dynamics in an operational railway embankment, 
Near Surf. Geophys., 12, 61-72, https://doi.org/10.3997/18730604.2013002, 2014.

Clemens, T., Hückinghaus, D., Liedl, R., and Sauter, M.: Simulation of the development of karst aquifers: role of the epikarst, Int. J. Earth Sci., 88, 157-162, 1999.

Clément, R., Descloitres, M., Günther, T., Ribolzi, O., and Legchenko, A.: Influence of shallow infiltration on time-lapse ERT: experience of advanced interpretation, C. R. Geosci., 341, 886-898, https://doi.org/10.1016/j.crte.2009.07.005, 2009.

Constable, S. C., Parker, R. L., and Constable, C. G.: Occam's inversion: a practical algorithm for generating smooth models from electromagnetic sounding data, Geophysics, 52, 289-300, 1987.

Coscia, I., Linde, N., Greenhalgh, S., Günther, T., and Green, A.: A filtering method to correct time-lapse 3-D ERT data and improve imaging of natural aquifer dynamics, J. Appl. Geophys., 80, 12-24, https://doi.org/10.1016/j.jappgeo.2011.12.015, 2012.

Dahlin, T. and Zhou, B.: A numerical comparison of 2-D resistivity imaging with 10 electrode arrays, Geophys. Prospect., 52, 379398, 2004

Dahlin, T. and Zhou, B.: Multiple-gradient array measurements for multichannel 2-D resistivity imaging, Near Surf. Geophys., 4, 113-123, 2006.

Descloitres, M., Ruiz, L., Sekhar, M., Legchenko, A., Braun, J.J., Mohan Kumar, M. S., and Subramanian, S.: Characterization of seasonal local recharge using electrical resistivity tomography and magnetic resonance sounding, Hydrol. Process., 22, 384394, https://doi.org/10.1002/hyp.6608, 2008.

Deville, S., Jacob, T., Chery, J., and Champollion, C.: On the impact of topography and building mask on time varying gravity due to local hydrology, Geophys. J. Int., 192, 82-93, https://doi.org/10.1093/gji/ggs007, 2012.

Ford, D. and Williams, P.: Karst Hydrogeology and Geomorphology, John Wiley and Sons Ltd., West Sussex, UK, 2007.

Ford, D. C. and Williams, P. W.: Karst Hydrogeology and Geomorphology, John Wiley and Sons, Chichester, UK, 2008.

Fores, B.: Gravimétrie et surveillance sismique pour la modélisation hydrologique en milieu karstique. Application au bassin du Durzon (Larzac, France), PhD thesis, Université de Montpellier, Montpellier, France, 2016.

Garré, S., Günther, T., Diels, J., and Vanderborght, J.: Evaluating experimental design of ERT for soil moisture monitoring in contour hedgerow intercropping systems, Vadose Zone J., 11, 412-424, https://doi.org/10.2136/vzj2011.0186, 2011.

Genty, D. and Deflandre, G.: Drip flow variations under a stalactite of the Pere Noel cave (Belgium). Evidence of seasonal variations and air pressure constraints, J. Hydrol., 211, 208-232, 1998.

Goldscheider, N. and Drew, D. P.: Methods in karst hydrogeology, Taylor and Francis, London, 2007.

Günther, T., Rücker, C., and Spitzer, K.: Three-dimensional modelling and inversion of dc resistivity data incorporating topography - II. Inversion, Geophys. J. Int., 166, 506-517, https://doi.org/10.1111/j.1365-246X.2006.03011.x, 2006.

Hartmann, A.: Putting the cat in the box: why our models should consider subsurface heterogeneity at all scales, Wiley Interdiscip. Rev. Water, 3, 478-486, https://doi.org/10.1002/wat2.1146, 2016.

Hartmann, A., Wagener, T., Rimmer, A., Lange, J., Brielmann, H., and Weiler, M.: Testing the realism of model structures to identify karst system processes using water quality and quantity signatures, Water Resour. Res., 49, 3345-3358, https://doi.org/10.1002/wrcr.20229, 2013.

Hartmann, A., Goldscheider, N., Wagener, T., Lange, J., and Weiler, M.: Karst water resources in a changing world: review of hydrological modeling approaches, Rev. Geophys., 52, 218 242, https://doi.org/10.1002/2013RG000443, 2014.

Hayley, K., Bentley, L. R., Gharibi, M., and Nightingale, M.: Low temperature dependence of electrical resistivity: implications for near surface geophysical monitoring, Geophys. Res. Lett., 34, L18402, https://doi.org/10.1029/2007GL031124, 2007.

Hunkeler, D. and Mudry, J.: Hydrogeochemical Methods, Taylor and Francis, available at: https://hal.archives-ouvertes.fr/ hal-00364736/ (last access: 2 May 2017), 2007.

Hunter, J. D.: Matplotlib: A 2-D Graphics Environment, Comput. Sci. Eng., 9, 90-95, https://doi.org/10.1109/MCSE.2007.55, 2007.

Kaufmann, O. and Deceuster, J.: Detection and mapping of ghostrock features in the Tournaisis area through geophysical methods - an overview, Geol. Belg., 17, 17-26, 2014.

Kaufmann, O., Bastin, C., Barcella, C., Watlet, A., and Van Ruymbeke, M.: Design and Calibration of a System for Monitoring Highly Variable Dripwater Flows in Caves, Mons, Belgique, 2016.

Kiraly, L.: Karstification and groundwater flow, Speleogenesis Evol. Karst Aquifers, 1, 155-192, 2003.

Klimchouk, A.: Towards defining, delimiting and classifying epikarst: its origin, processes and variants of geomorphic evolution, Speleogenesis Evol. Karst Aquifers, 2, 1-13, 2004.

Kuras, O., Pritchard, J. D., Meldrum, P. I., Chambers, J. E., Wilkinson, P. B., Ogilvy, R. D., and Wealthall, G. P.: Monitoring hydraulic processes with automated time-lapse electrical resistivity tomography (ALERT), C. R. Geosci., 341, 868-885, 2009.

Kuras, O., Wilkinson, P. B., Meldrum, P. I., Oxby, L. S., Uhlemann, S., Chambers, J. E., Binley, A., Graham, J., Smith, N. T., and Atherton, N.: Geoelectrical monitoring of simulated subsurface leakage to support high-hazard nuclear decommissioning at the Sellafield Site, UK, Sci. Total Environ., 566, 350-359, 2016.

LaBrecque, D. J., Ramirez, A. L., Daily, W. D., Binley, A. M., and Schima, S. A.: ERT monitoring of environmental remediation processes, Meas. Sci. Technol., 7, 375, 1996a.

LaBrecque, D. J., Miletto, M., Daily, W., Ramirez, A., and Owen, E.: The effects of noise on Occam's inversion of resistivity tomography data, 1996b.

Lange, J., Arbel, Y., Grodek, T., and Greenbaum, N.: Water percolation process studies in a Mediterranean karst area, Hydrol. Process., 24, 1866-1879, https://doi.org/10.1002/hyp.7624, 2010.

Lecocq, T., Longuevergne, L., Anette Pedersen, H., Brenguier, F., and Stammler, K.: Monitoring ground water storage at mesoscale using seismic noise: 30 years of continuous observation and thermo-elastic and hydrological modeling, Sci. Rep.-UK, 7, 14241, https://doi.org/10.1038/s41598-017-14468-9, 2017.

Mahmud, K., Mariethoz, G., Baker, A., Treble, P. C., Markowska, M., and McGuire, E.: Estimation of deep infiltration in unsaturated limestone environments using cave lidar and drip count data, Hydrol. Earth Syst. Sci., 20, 359-373, https://doi.org/10.5194/hess-20-359-2016, 2016.

Mahmud, K., Mariethoz, G., Baker, A., and Treble, P. C.: Hydrological characterization of cave drip waters in a porous limestone: 
Golgotha Cave, Western Australia, Hydrol. Earth Syst. Sci., 22, 977-988, https://doi.org/10.5194/hess-22-977-2018, 2018.

Mangin, A.: Contribution à l'étude hydrodynamique des aquifères karstiques, PhD thesis, Université de Dijon, Dijon, France, 1975.

Marion, J.-M., Mottequin, B., Barchy, L., Blockmans, S., and Dumoulin, V.: Contexte géologique et structural de la région de Rochefort (Synclinorium de Dinant, Belgique), Geol. Surv. Belg. Prof. Pap., 2011, 27-38, 2011.

McKinney, W.: pandas: a foundational Python library for data analysis and statistics, Python High Perform. Sci. Comput., 1, 1-9, 2011.

Meyerhoff, S. B., Karaoulis, M., Fiebig, F., Maxwell, R. M., Revil, A., Martin, J. B., and Graham, W. D.: Visualization of conduit-matrix conductivity differences in a karst aquifer using time-lapse electrical resistivity: ERT time-lapse of karst conductivity, Geophys. Res. Lett., 39, L24401, https://doi.org/10.1029/2012GL053933, 2012.

Michot, D., Benderitter, Y., Dorigny, A., Nicoullaud, B., King, D., and Tabbagh, A.: Spatial and temporal monitoring of soil water content with an irrigated corn crop cover using surface electrical resistivity tomography, Water Resour. Res., 39, 1138, https://doi.org/10.1029/2002WR001581, 2003.

Parasnis, D. S.: Reciprocity theorems in geoelectric and geoelectromagnetic work, Geoexploration, 25, 177-198, 1988.

Perrin, J., Jeannin, P.-Y., and Zwahlen, F.: Epikarst storage in a karst aquifer: a conceptual model based on isotopic data, Milandre test site, Switzerland, J. Hydrol., 279, 106-124, https://doi.org/10.1016/S0022-1694(03)00171-9, 2003.

Pipan, T. and Culver, D. C.: Estimating biodiversity in the epikarstic zone of a West Virginia cave, J. Cave Karst Stud., 67, 103-109, 2005.

Pirson, S., Spagna, P., Baele, J.-M., Damblon, F., Gerrienne, P., Vanbrabant, Y., and Yans, J.: An overview of the geology of Belgium, Mem. Geol. Surv. Belg., 55, 5-25, 2008.

Poulain, A., Rochez, G., and Hallet, V.: Caractérisation hydrogéologique du réseau de la Lomme souterraine, in: Atlas du Karst Wallon: Bassin de la Lesse Calestienne, Service Public de Wallonie, Jambes, 2015a.

Poulain, A., Rochez, G., Bonniver, I., and Hallet, V.: Stalactite drip-water monitoring and tracer tests approach to assess hydrogeologic behavior of karst vadose zone: case study of Han-sur-Lesse (Belgium), Environ. Earth Sci., 74, 7685-7697, https://doi.org/10.1007/s12665-015-4696-9, 2015b.

Poulain, A., Watlet, A., Kaufmann, O., Van Camp, M., Rochez, G., Deleu, R., Quinif, Y., and Hallet, V.: Assessment of groundwater recharge processes through karst vadose zone by cave percolation monitoring, Hydrol. Process., in review, 2018.

Pronk, M., Goldscheider, N., Zopfi, J., and Zwahlen, F.: Percolation and Particle Transport in the Unsaturated Zone of a Karst Aquifer, Ground Water, 47, 361-369, https://doi.org/10.1111/j.1745-6584.2008.00509.x, 2009.

Quinif, Y., Van Ruymbeke, M., Camelbeek, T., and Vandycke, S.: Les failles actives de la Grotte de Rocheforrt (Ardenne, Belgique) sont-elles sismogéniques? Installation d'un laboratoire souterrain, Aardkundige Mededelingen, 8, 153-156, 1997.

Revil, A., Cathles, L. M., Losh, S., and Nunn, J. A.: Electrical conductivity in shaly sands with geophysical applications, J. Geophys. Res., 103, 925-23, 1998.
Revil, A., Karaoulis, M., Johnson, T., and Kemna, A.: Review: some low-frequency electrical methods for subsurface characterization and monitoring in hydrogeology, Hydrogeol. J., 20, 617-658, https://doi.org/10.1007/s10040-011-0819-x, 2012.

Rhoades, J. D., Raats, P. A. C., and Prather, R. J.: Effects of liquidphase electrical conductivity, water content, and surface conductivity on bulk soil electrical conductivity, Soil Sci. Soc. Am. J., 40, 651-655, 1976.

Robert, T., Caterina, D., Deceuster, J., Kaufmann, O., and Nguyen, F.: A salt tracer test monitored with surface ERT to detect preferential flow and transport paths in fractured/karstified limestones, Geophysics, 77, 55-67, https://doi.org/10.1190/geo2011-0313.1, 2012.

Rücker, C., Günther, T., and Spitzer, K.: Three-dimensional modelling and inversion of dc resistivity data incorporating topography - I. modelling, Geophys. J. Int., 166, 495-505, https://doi.org/10.1111/j.1365-246X.2006.03010.x, 2006.

Rücker, C., Günther, T., and Wagner, F. M.: pyGIMLi: an open-source library for modelling and inversion in geophysics, Comput. Geosci., 109, 106-123, https://doi.org/10.1016/j.cageo.2017.07.011, 2017.

Samouëlian, A., Cousin, I., Tabbagh, A., Bruand, A., and Richard, G.: Electrical resistivity survey in soil science: a review, Soil Till. Res., 83, 173-193, https://doi.org/10.1016/j.still.2004.10.004, 2005.

Sheffer, N. A., Cohen, M., Morin, E., Grodek, T., Gimburg, A., Magal, E., Gvirtzman, H., Nied, M., Isele, D., and Frumkin, A.: Integrated cave drip monitoring for epikarst recharge estimation in a dry Mediterranean area, Sif Cave, Israel, Hydrol. Process., 25, 2837-2845, https://doi.org/10.1002/hyp.8046, 2011.

Sket, B., Trontelj, P., and Zagar, C.: Speleobiological characterization of the epikarst and its hydrological eighborhood: its role in dispersion of biota, its ecology and vulnerability, in: Epikarst: Proceedings of the Symposium held October 1 through 4, 2003, Shepherdstown, West Virginia, USA, edited by: Jones, W. K., Culver, D. C., and Herman, J. S., Karst Waters Institute Special Publication 9, Charles Town, West Virginia, USA, 104-113, 2004.

Smart, P. L. and Friederich, H.: Water movement and storage in the unsaturated zone of a maturely karstified carbonate aquifer, Mendip Hills, England, in: Proceedings of the Environmental Problems in Karst Terranes and Their Solutions, National Water Well Association, Dublin, Ohio, USA, 59-87, 1987.

Supper, R., Ottowitz, D., Jochum, B., Kim, J.-H., Römer, A., Baron, I., Pfeiler, S., Lovisolo, M., Gruber, S., and Vecchiotti, F.: Geoelectrical monitoring: an innovative method to supplement landslide surveillance and early warning, Near Surf. Geophys., 12, 133-150, 2014.

Triantafyllou, A., Watlet, A., Kaufmann, O., and Le Mouelic, S.: Exploring structures of the Rochefort Cave (Belgium) with 3D models from LIDAR scans and UAV photoscans, AGU Fall Meeting 2016, 12-16 December 2016, San Francisco, USA, T41A-2897, 2016.

Uhlemann, S., Smith, A., Chambers, J., Dixon, N., Dijkstra, T., Haslam, E., Meldrum, P., Merritt, A., Gunn, D., and Mackay, J.: Assessment of ground-based monitoring techniques applied to landslide investigations, Geomorphology, 253, 438-451, https://doi.org/10.1016/j.geomorph.2015.10.027, 2016a. 
Uhlemann, S. S., Sorensen, J. P. R., House, A. R., Wilkinson, P. B., Roberts, C., Gooddy, D. C., Binley, A. M., and Chambers, J. E.: Integrated time-lapse geoelectrical imaging of wetland Hydrol. Process., Water Resour. Res., 52, 1607-1625, https://doi.org/10.1002/2015WR017932, 2016b.

Valois, R.: Caractérisation structurale de morphologies karstiques superficielles et suivi temporel de l'infiltration à l'aide des méthodes électriques et sismiques, $\mathrm{PhD}$ thesis, Université Pierre et Marie Curie-Paris VI, Paris, France, 2011.

Van Camp, M., Meus, P., Quinif, Y., Kaufmann, O., Ruymbeke, M., Vandiepenbeck, M., and Camelbeeck, T.: Karst aquifer investigation using absolute gravity, Eos Trans. Am. Geophys. Union, 87, 298-298, https://doi.org/10.1029/2006EO300005, 2006.

Vandycke, S. and Quinif, Y.: Recent active faults in Belgian Ardenne revealed in Rochefort Karstic network (Namur province, Belgium), Nord. J. Geosci., 80, 297-304, 2001.

Voisin, C., Garambois, S., Massey, C., and Brossier, R.: Seismic noise monitoring of the water table in a deep-seated, slowmoving landslide, Interpretation, 4, SJ67-SJ76, 2016.

Watlet, A., Kaufmann, O., Triantafyllou, A., Poulain, A., Chambers, J. E., Meldrum, P. I., Wilkinson, P. B., Hallet, V., Quinif, Y., Van Ruymbeke, M., and Van Camp, M.: Data and results for manuscript "Imaging groundwater infiltration dynamics in karst vadose zone with long-term ERT monitoring", Data Set Zenodo, https://doi.org/10.5281/zenodo.1158631, 2018a.
Watlet, A., Van Camp, M., Francis, O., Poulain, A., Rochez, G., Hallet, V., and Kaufmann, O.: Gravity changes associated with underground flood events used as proxies for characterizing karst voids, Water Resour. Res., submitted, 2018b.

Waxman, M. H. and Smits, L. J. M.: Electrical conductivities in oilbearing shaly sands, Soc. Petrol. Eng. J., 8, 107-122, 1968.

White, W. B.: Karst hydrology: recent developments and open questions, Eng. Geol., 65, 85-105, 2002.

Wilkinson, P. B., Loke, M. H., Meldrum, P. I., Chambers, J. E., Kuras, O., Gunn, D. A., and Ogilvy, R. D.: Practical aspects of applied optimized survey design for electrical resistivity tomography, Geophys. J. Int., 189, 428-440, 2012.

Willems, L. and Ek, C.: Le système karstique de la Lomme. Quelques points d'observation remarquable, Geol. Surv. Belg. Prof. Pap., 2011, 3-10, 2011.

Williams, P. W.: The role of the epikarst in karst and cave hydrogeology: a review, Int. J. Speleol., 37, 1-10, 2008.

$\mathrm{Xu}$, S., Sirieix, C., Riss, J., and Malaurent, P.: A clustering approach applied to time-lapse ERT interpretation - case study of Lascaux cave, J. Appl. Geophys., 144, 115-124, https://doi.org/10.1016/j.jappgeo.2017.07.006, 2017. 